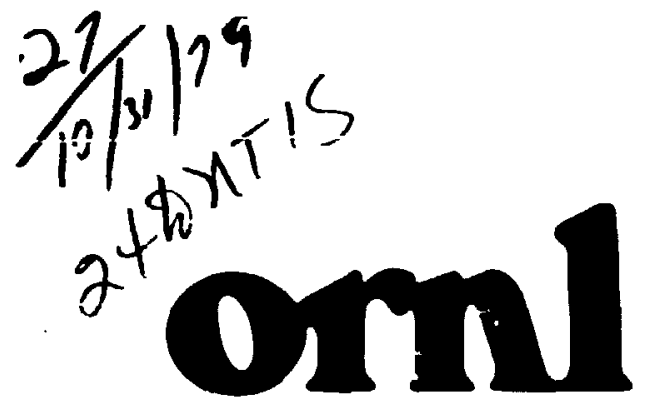

OAK

RIDGE

NATIONAL

LABORATORY

UNION

CARBIDE

\title{
Bibliography of Articles on Radioactive Waste From 1973-1978
}

\author{
Lois Martin Bronfman
}


Contract No. W-7405-eng-26

BIBLIOGRAPHY OF ARTICLES ON RADIOACTIVE WASTE

From 1973-1978

By

Lois Martin Bronfman

with the assistance of

Emily Passino, Elaine G. Llevellyn and Polly Parnell

Energy Division

Prepared for

office of Integrated Assessment and Policy Analyois Health and Safety Research Diviston

Oak Ridge National Laboratory

Date Published - October 1979

OAK RIDGE NATIONAL L"BORATORY

Oak Ridge, Tennessee 37830

operated by

UNION CARBIDE CORPORATION

for the

DEPARTMENT OF ENERGY

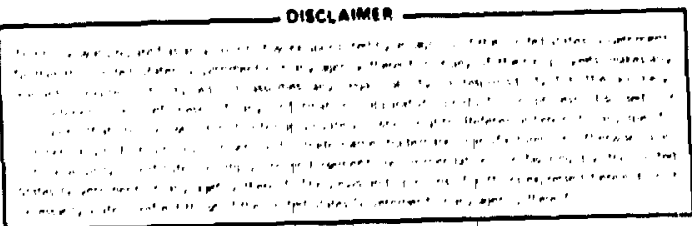




\title{
BIBLIOGRAPHY OF ARTICLES ON RADIOACTIVE WASTE from 1973-78
}

By

\author{
Lois Martin Bronfman \\ with the assistance of \\ Emily Passino \\ Elaine G. Llewellyn \\ Polly Purnell
}

This bibllography is a supplement to a study on the content of public information on radioactive waste. The articles were collected for the period 1973-78, a time in which radioactive waste management emerged as an important policy issue confronting the nation.

The bibliography distinguishes between information sources based on the different interests which the audiences to the pub-ications might represent in the controversy over radioactive vaste management. The major categorfes chosen were: national press, Al; general scientific press, A2; nuclear scientific fress, A3; nuclear industry/utility, A4; environmental press, A5; and local press, A6.

The search for articles on radioactive vaste involved several steps. For discussions of radioactive waste in popular sources the Reader's Guide to Periodical Literature as well as the New York Times Data Bank were consulted. All articles mentioning radioactive waste and indexed for the period between January 1973 to June 1978 were iricluded in the biblfography. To search out articles in the more specialized information sources (e.g., Nuclear News, Science, Critical Mass), the Central Research Library and the Energy and Environmental Response Center Library at Oak Ridge National Laboratory as well as the personal libraries of some of ORNL's staff were used. A spectrum of journals and newsletters from each specialized source was sought. Each journal selected was searched for radioactive waste articles appearing during the 1973 through 1978 period. In addition the former Office of Waste Isolation of the Department of Energy made avallable its newspaper clippings from local newspapers in the states in which OWI was taking steps to determine the suitability of selected sites for hosting a radioactive waste repository. In this bibliography articles which appeared in local newspapers in Michigan and Loufstana were included for the years 1976 and 1977, a time of considerable controversy within these states over the OWI's actions. 
NATTONAL PRESS 
M

Q n beclear waste be storen?

peusweet. F. 56: 1974, Woutenber 10

The Great welerer wate

Ti E. F. 36-37: 1975. Deceaber

Th- Iystege tiagter

buswet. Ue, 45: 1976, Decee oer 20

The belear cobte

-iee, p. 30: i970. Agril is

inclear Powe planes: Dow Iistrp

7. 5. weves borld Eeport. P. 55-56: 1976, warch 29

meloar second poughes

bev Tork Tines: 1076. Septenber 25

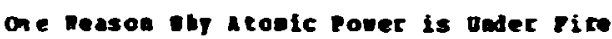

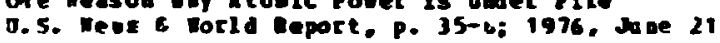

Putting Crakes on the last Breeder: 3 . Carter:s Enlicy on Fiotoni be Eejcliog

T10. 109. 57: 1977. Apr 11 18

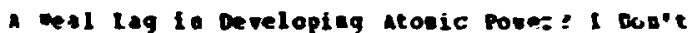
aree

ח.5. yeus o sor ld aefore, p. 30-11; 1975 . se freaber $\mathrm{C}$

Mifiactive daste Duilds np in misos

The meo Port Plues: 1975, October 1h

Peport opeivistic ca neole Dasen

the ver rork Tiees. p. 15; 1976, hat 11

Sed hat seceive Meclear wastes

the Dee rork ribes: 1976. Mprll 15

Too many gags la weclear easket?

The Chrietila sctence honteor, p. 10: 1974.

sp preaber 25

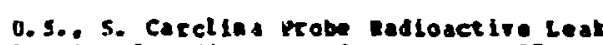
Los ingeles fiese, fo 2, pact I: 1976, Hoys

The Dandly Dilewne of meclear mastes

eusiness Deet, p. pe-7i: 1975, march

wer Mlares Aront oif poclear Destes

posinace Deek, P. 17-1A: 1976, Pabruary 2

preqrad 10 conesin muclear knowhor

hbsiness wak, p. 31: 1977, Apr 11 11

Pravantinq: ui tease vartare

fortes, F. i15, 149: 1975, Pebruart

a Pugh tc Ground hezerde us lit cargo

husinase reek, p. 17; 1970, April 20

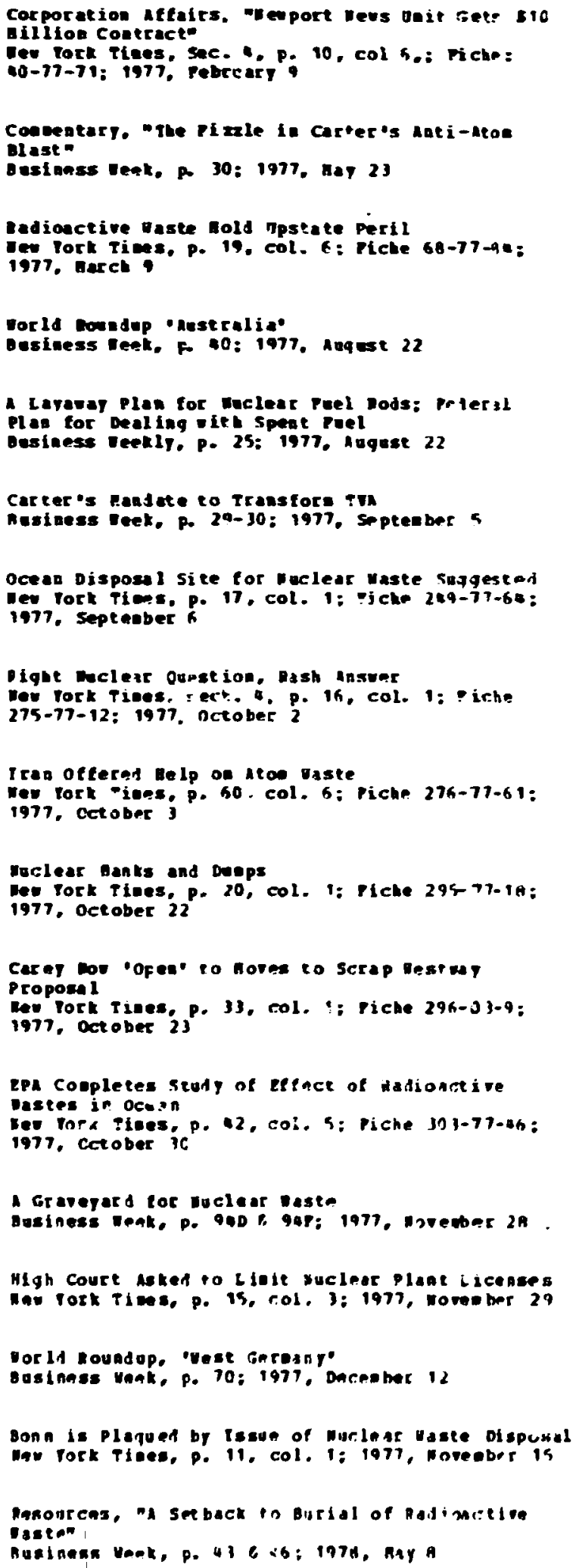


Eist reteans Sper acon pover with Do oped Pablic Cppositica to Torl tives, p. 6, col. 5: Piche 150-70-55: 1978. $\operatorname{tar} 30$

- tropolitae briefs, "Bee on melear mates" - tork tiees, sec. 2, p. 3, col. ": Tiche 199-78-76: 1778, April t9

iptertor bisfutes "unsafe' zharges on Meopic naste sey tort pies, f. 29, col. I: Plele 127-76-47: 1978, 7ะพุ 7

rnverneent Protlees ie Findiag ofiposal sites for

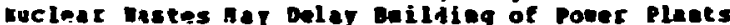
se tark tlees. p. 34. col. 1: piche 106-09-90: 1976. April is

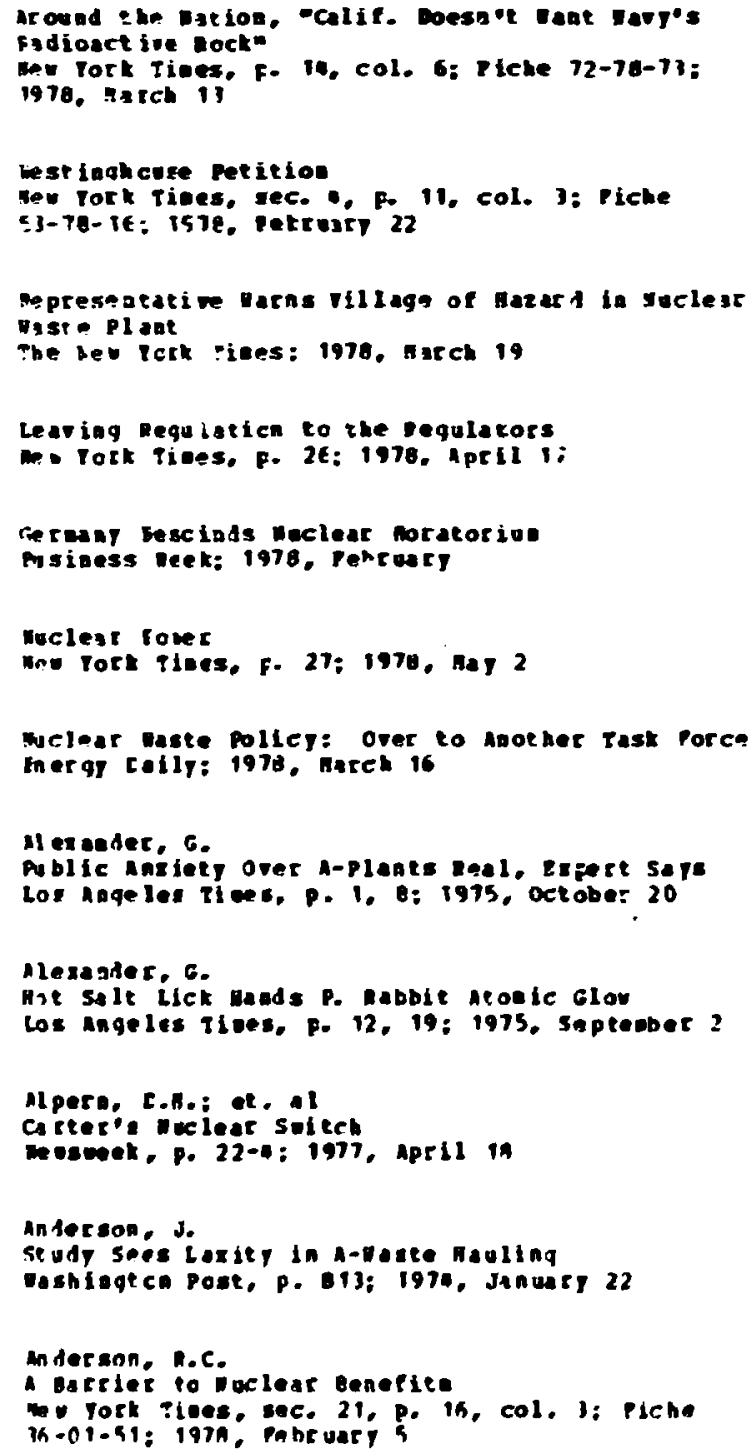


collke, L, $\mathrm{t}$

Humen toli and welear Toctinologp

Cutrent. 161. ithe: 1974, April

El iger . C.: Farist a

M.5. Eucleat Pever nor to on The Irong traek

cosiness teek, P. 192- 3: 1977, April 10

Faber. $\mathrm{ll}$.

Site coard Afprover opstate htos plant

wev Tork rises, sect. 2, P. 7, col. I: Piete

342-77-ha: 1577, Decenber

Pa reer. $t$.

Avesobe froble: of Hoclear ilestes

peeder's Digsst. P. A3-7: 1974, angest

Pollevs, :-

Consecticet Bill zecote Aton Deste shiptents

The Eet Tork Tibes: 1976, March id

Prevdenteit. I.

meleet iJuni stirs pebate is zorop

instingtce Past, P. A-12: 1976, Angust 14

tapt. 1 .

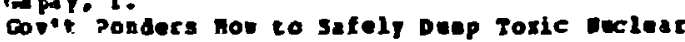

wa nete

wil sereet Jourasl. p. 1. 15: 1976, Jely 26

Gillette,

Pising rangers of aeonfe lastes

I.5. Heiz C Norid Meport. P. 30-32: 1973,

sopteber 10

Gi llette.

ne Danger of meclear progress. Wucleac teste

The weo tork times: 1975. Mey it

Gorean, J.

Cisposing of eclear enste

Current, 181, 38-1,1; 1976, narch

Gunne, F.: Bishep, J.. Jr.: Michaed, S.6.

mot Safe is noclear power

necsmek. P. 70-75: 1976. April 12

Heitch, $A$.

pollow-af on ewe wars, "htonle Leetage"

- Tork Iinas. P. 37, col. 5; Plehe 219-02-06;

1977, Mugese 7

mandrin, I.

The weclear pouer plest coserones

The Lod Algales Itees, Soction IT. P. 1, A-5:

1975, not?

marp. n.

Connecticut naks Hor to spend onerpecent surplus

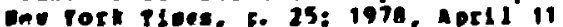

ni $11,6$.

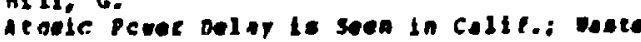

Preblec citen

mav rock Tíes, F. 11, col. 1: Plem 212-31-19. 1977. Joly 39 ail1, 5

coust Legislatere Apporeat ly Dooe Inclear plant cer Tock tims, p. 13, col. T: Piche 104-78-67: 1978. April 10

nill. 6.

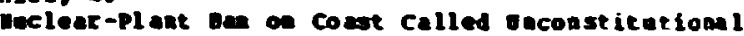
Eet tork Tiuns, P. 10, col. 3: Pletre 122-78- 36:

1978. Hat 2

iill. C.

penel cuks licensing sespension

we Port Time. p. 10; 1970, MaI 2

aili, 6

Califoroil Dard ron't Gi perait for the nuclear tangy Profece

Det Jort Tibes, sec. 2, p. 2, col. 2: piche 32-79-79; 1970, Pebreary;

Bonse. I. E.

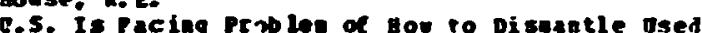
ieactors.

Tie nall street Joural: 1977, October 12.

Iednesday

Moward, J.6.

Leters, "Huclear Deflections"

tour tions, p. 28, col. 5; Plcbe 136-77-325: 1977. May 16

Iocebie. T.t.

0.5. Maclear Iudeatry sees a turent :o Exports in Bil

Mee rork Tims, ace. A, p. 1, col, A: Cotrection on: Les rotk qices, see. 2, p. 1. Gol. 6: 197A, Pebruary 15

roing, 8.

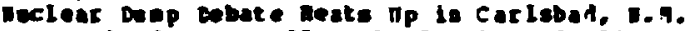

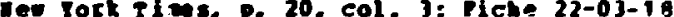

1978, Jagert 22

Janson, D.

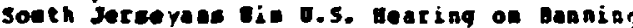

Atonfe inte stor sge

Dev Jork ILees, sec. 2, p. In, col, I: Picbe

76-78-83: 1978, sareh it

KLeivan, 1.

Pletonies Dangers

Dew Iork pless: 1976, septenber 20

Nalque, J.

Woclear Power: Tha angel of Deet

corporate esasswer, Ineart, p. 34-3D; 1971, oct obet

Treft. J.

Certer". Forelga polley hothod

vasulagton Pose, P. A-21: 1977, March is

Lenogetes, 1.J.

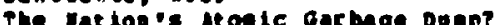

The Daefonel overom: 1973, Fohruery?

Lanoverta,

Hot reter

the Motionel oberenc, p. 9; 1979, January 
thetcher. 6 .

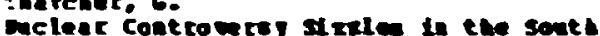

christiel sclence Gonter. po 18-19: 1976. coreeser 26

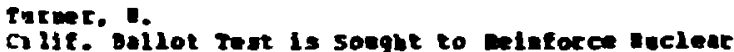
Controis

tov rork Ilves. F. A, col. 5: piche 21-70-61:

Ti 11 . E. S.

The sireas sieg trou Picenee creet

progresstoe. P. a-52: 1974. April

E. Id. E.t.

O. S. Gav cuerene we tork city ban on buclear

Treffic

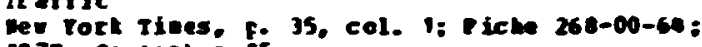

1977. Sertenter 25

Da 1d. B.t.

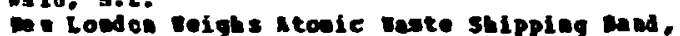

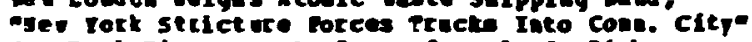
nes rort ribes, sect. 2, p 3, col. I: Plicte

7-70-56: is7., iptil it

D 1d, B. I.

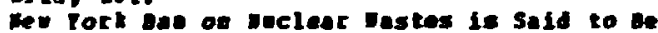

iccopted ty 8.S.

weo fort Ilees. p. 27, col. S; Piche 5-78-30:

1978. April 5

1ald. A.t.

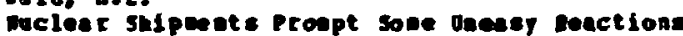

- rort Tines. ec. 23, p. 1, col. 3: Viche

71-03-56: 1978: wareh 12

vald, R.t.

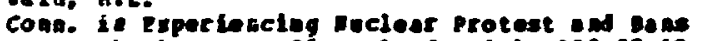
we rork ribes, $p$. 35. col. 1: Flebe 120-07-62: 1970, April 30

1010. 3.3.

Lecters:- ingeterical Duelear seenclos"

wo rort if es. p. 22, col. 3: Plebe 342-n-25:

1977. Deceober

Tebeter.

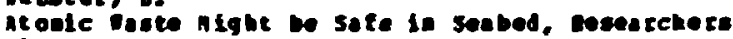

Pt nd

mo ta: a riees, fo 24, col. 1: Plew 62-77-5e:

1977. Aarch?

isenen, 3.t.

stee Aces on on odjocetive prasho

we tort riees. p. is, col. 5: piets 114-7e-26:

1979, Apsil 29

thite. D.

melaer pouer: special noo societp surmt

ic. Socleti: ist7, hareh 31

Heker, ?.

m Pork lives, p. 39, col. 5: Flebe 270-77-15:

1977, sopteober 27

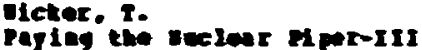

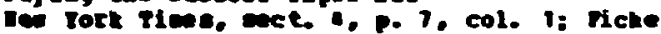

$275-77-92$ : 1977, octower 2

Icker. T.

nare vicier bohet

Des Tork Hises. p. 33, col. 5: Hehe 312-77-44:

1977. Corevber 8

Tieker, T.

reacting igal est beetore

Dew tork riess. 29. col, 5: piche 69-78-78;

1970. Aurch 10

112. C.P.

veclest powr as a social Isere

vestegton pore. p. A-19: 1976. Jane

VLleon. R.

lettera, ato Iraneport u-lear vastio

Dow tort riees, P. 32, col. 3; Piele 30n-77-25;

1977. Boreeter 
GENERAL SCIENTIFIC PRESS 


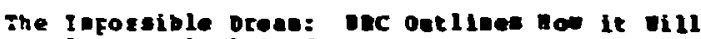
the velop tegelaticas for uste

The seergy Dat IY. P. 2-3: 1976. Woverber 3

Career on Declear Powr: befer beprocessing

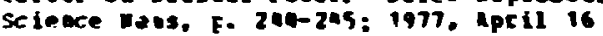

Pire is The Ashes

scienelfic peericae. P. 56-62: 1976. Javery

Fossil Foel palleut

setence cigest. 75, 69-70: 1974, Jane

WRC to DFiate vaste had ysis

ir outc laf estrial Pordefimp: 1976, Septeaber

Techwolog7. Experts all Ger gadioace fre vaste ri sposal"

Chesical and engibeeriog vers, p. 21-23: 1976. hojost 2

Ganges orged in Joclear uste Pegelations Cheofcal end engincering weve, f. 20-21: 1976 , sept eater 20

world woclear vaste seandar ts called for cheoical sad raqineering Eevs, F. 5: 1976, July 19

Wuclear enste risposal Plan gets unc ora Cheolcal and paqiaeerinq Veus, p. 6; 1977 , datery 10

ER CA's plans fCr woclear iastes lapped Cherical and engineering neus, p. 7; 1977, Janeary it

Nuclear thergy

Enqinepring sclence c rechnology: 1977, Pobrury

welaar bedirection

Cheofical Heak, p. 21: 1977, April 20

Wore fer Spent Toal Dods

Cheajtil veet, p. 69: 1977, April 27

sedy finds Noclear bate rechnolojy soond Cbesical $c$ zogineeriag Feus, 55, P. 17: 1977, As

Lisers Heduce raclese naste

Caedistr, 50, 24-25; 1977, hay

science 6 the citizen

Srience: 1577, June

Wational h-Waxte Plan rooing: Will speet plane

Conetracelion

ta gineer ing Mers-pecord, 199, 12; 1971, Angose 1h

Technology. Sites soughe for waclad uanee

Cisposal

Chesical and englnencing wavs, p. 23: 1976,

augune?
GA) orges Solution to Wecleat unte disposal cheicel s teginering Bevs, P. 6: 1977 .

septeaber 19

White bouse Incleat Daste plat

inqineerting vews lecord: 1977, October

0.S. to protide storage for spont Vuclear pel

cheofcal and tagineering wos. P. K: 1977.

octobaz 20

- Interio" Policy for Spene Puclear Puel

Sclence mens, 112, p. 278 : 1977, october 79

- Mired verfict on mac velear laste Dorupentary

Scier.ce, 19a, 1232-1233: 1977, Decenber 23

Ohite Mouse Uuclear waste plan ray onleash Plant

constract ion

bigineering wews-record, 199, 12; 1977, Jetoher 27

Wuclear projects Threatenes hy Haste Disposal

Del mana

Eoqineering veus mecord, 200, 3: 1376, J, nuary 19

ntonic haste

Science: 1978, mach 10

Abel soe, P. 月.

Glanorous Doclear Pission

Scinnce, P. 279: 1976, Jol I 23

Auq ino, E.E.

Migh-lerel and Loog-lifed padioactive maste

Disposal

Sclench, 19月, 895-90; 1977, necenber 2

Debbingtog, M.P.

Meprocessing of wuclsar pols

Sciediftc huerican, 235, 30-41: 1976, Decenber

Bot be, H. A.

The necassity of Fission power

Sclenpific nerticio, p. 21-31; 1976, January

Dofley. P.n.

Radiactive vate site sench Gets into bep yater Science, p. 361: 1975, Octoher 24

Bul 1. C.

Radioareive Iaste Disponl

Science, 189, 596-597, 659. Leter: 1975, Auguet

22

Career, h.J.

Mocleas Inithatives: Tu alles Disagrea on

heasing of jefene

science, p. 811-812; 1276, Norenber 19

Cartar, t...3.

seate scientific advisore: The effort in wichigan

sclence, p. 921, 912: 1976, Movenher i6

Carter, t. J.

lest vallep: The oustion is uhare Does Buct

3top on Nuclaer Wates?

Scionce, 195, 1304-1304; 1971, Nareh 25 
Cartex. L.J.

Paliactive uastes: sone orgent onflaishem Quisus: 5

sciepe. p. 661: 1971, petruary 10

Carter. 1.J.

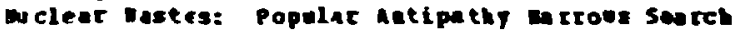

for nisposal jices.

Science, 157, 1265-1266; 1917, Soptenber 21

Cacter, 1.J.

Congressicenl conoittees poder ohother to clre

st ates idglt of reto orer Radionctive waste

peposteortes

srience. 200, 1136-1131; 1978, Jane 9

Carter. I.J.

oriefirq. "trouble the in ter Texico for nuclear waste Discosil

se ieece. 199, 1050-1051: 19:3, Bat ch

carter, L.J.

The padicactive maste Inrentory

stience. F. 197: 1977, Pebcaly 18

Carter, L-J.

Mucleet gafes: The Science he Gpologic jisposal

son as leat

Scienco. 2C0, 1135-1131: 1978, Jone

Caned, B.t.

In pace s of the Nuclear toeryl I adustry on Huna

Realt in ond satet?

American Scientist, 64, 550-559; 1976 ,

sefpentier-catober

Cohen, R.L.

The Oisposil of Radiosctive vastes Pron Pission

meactors

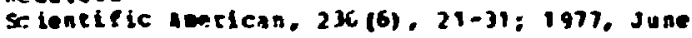

de narsily, G.: Letoux, E.: Barbreav, A.: Margot.

r.

wuclest parte ci uposel: cen the Geologist

Guranete laclint $t$ on

science, 157(4303): 1977, Auguat 5

wioa, i.c.

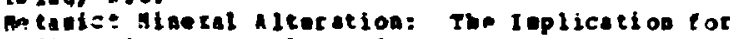

Padloartive aste elsposal

srtence, F. 136-1337; In7h, Jope 25

Pirney, r.

oo inows trotleo: whe to do with mationctive

uate

Seithsenieb, 5, 20-7: 1974, Apr 11

Pried, S. and h. R. Peiodeden

Ma tention of plutoni us and hoer icun by hock

sctence, 196, 1097-1084; 1977, June?

Gillnsky, I.

Platonias, proliteration, and policy

Technolcal Reviev, p. 58-65; 1971, Pebruary

Gillepte. B.

on Inholing pluecalue: Oas moes Long senty

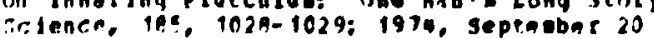

Gllete.

Meclear reel seprocensing: GP's balky pleet

pases stortege

Sctunce, 195, 770-771: 1974, Meguse 10

Gillete.

WDa Shelres a Dclear ase Prograe

scimce. P. 305; 1975. April 75

Gilete. n.

Senctors ant Gerean, Prench vicleer sales

sciece. p 1193: 1975. June 2C

Gillette, $\mathrm{E}$.

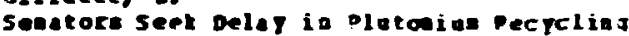

science. P. 128: 1970, Detoset 11

Gillete, 1.

Transiene" noclear workers: a spacinal case for

seantards: wecleer Poel ser aites puployees

Science, 196, 125-127, 129; 1970, October

cilleter,

Pectciling Pleconien: Ixc proposes a secont Look

Science, 19a, d16, 075: 1975, Mat 23

Gillerte.

Ploconlueviching and biting for atverse cftects

science. 185, 1100-1103: 1979. Septesber 21

Gillete.

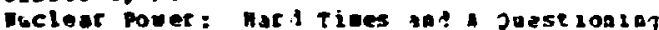

Congrese

sctence. P. 1058-1062: 1975, Anch 21

Gillete. P

Pluconios (I): Questions of Healph it sev

Induety

Sctence, 195, 1027-1012: 1970, Septaber 23

Giletes,

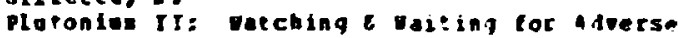
effects

Scionce, 185, 1900-1143: 1970, Sop., 27

Hageonit. to

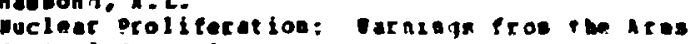
Conerol coseunity

sctence, , 126-128: 1976, July?

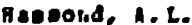

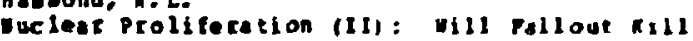

Donesete pecycie?

Science. P. 217-218; 1976, Julf 16

Havkes,

welear bastes seyeie vast rotouns

sclence, p. 962-963: 1977, sereb 11

Haukes, M.

science in zurope: Beitish ponder feprecenging

Plans

Scionce, p. 31-38: 1977, January?

Holdan $\mathrm{C}$

Ken fucky inheppy litb heor Doep

science, 199, 36: 197a, Innuary, 


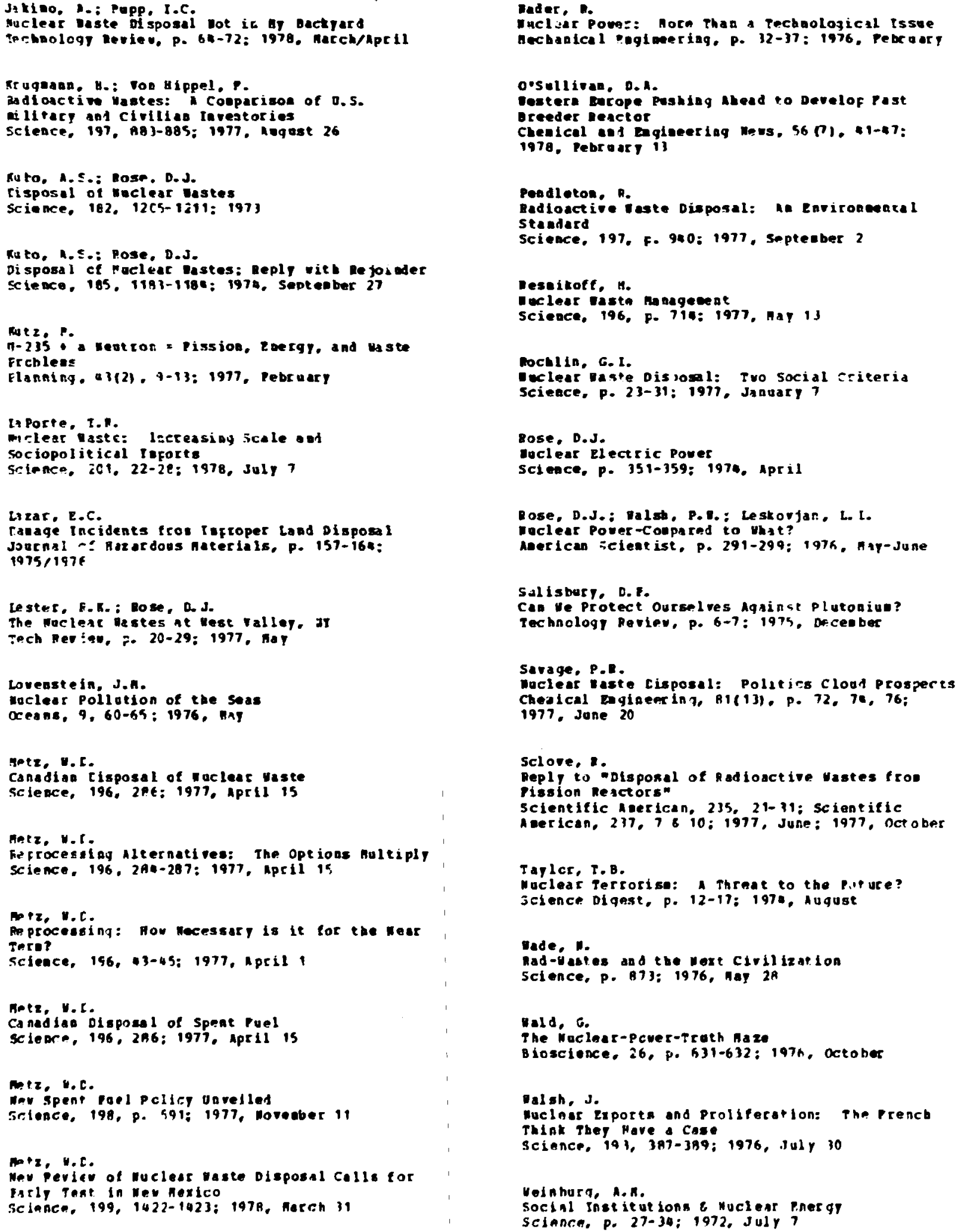


ur ane-Fduards, A.B.

any the vuclear option is oot fet endy for

Pull-scale ajoption by caeala

sripnce loces, 9, 13-17: 1976, hugest

2ollet, I.J.

The Dispesal of suclenr Daste

calitorola Gfology. F- 79-87: 1973, April 
NUCLEAR SCIENTIFIC PRESS 


\section{BLANK PAGE}




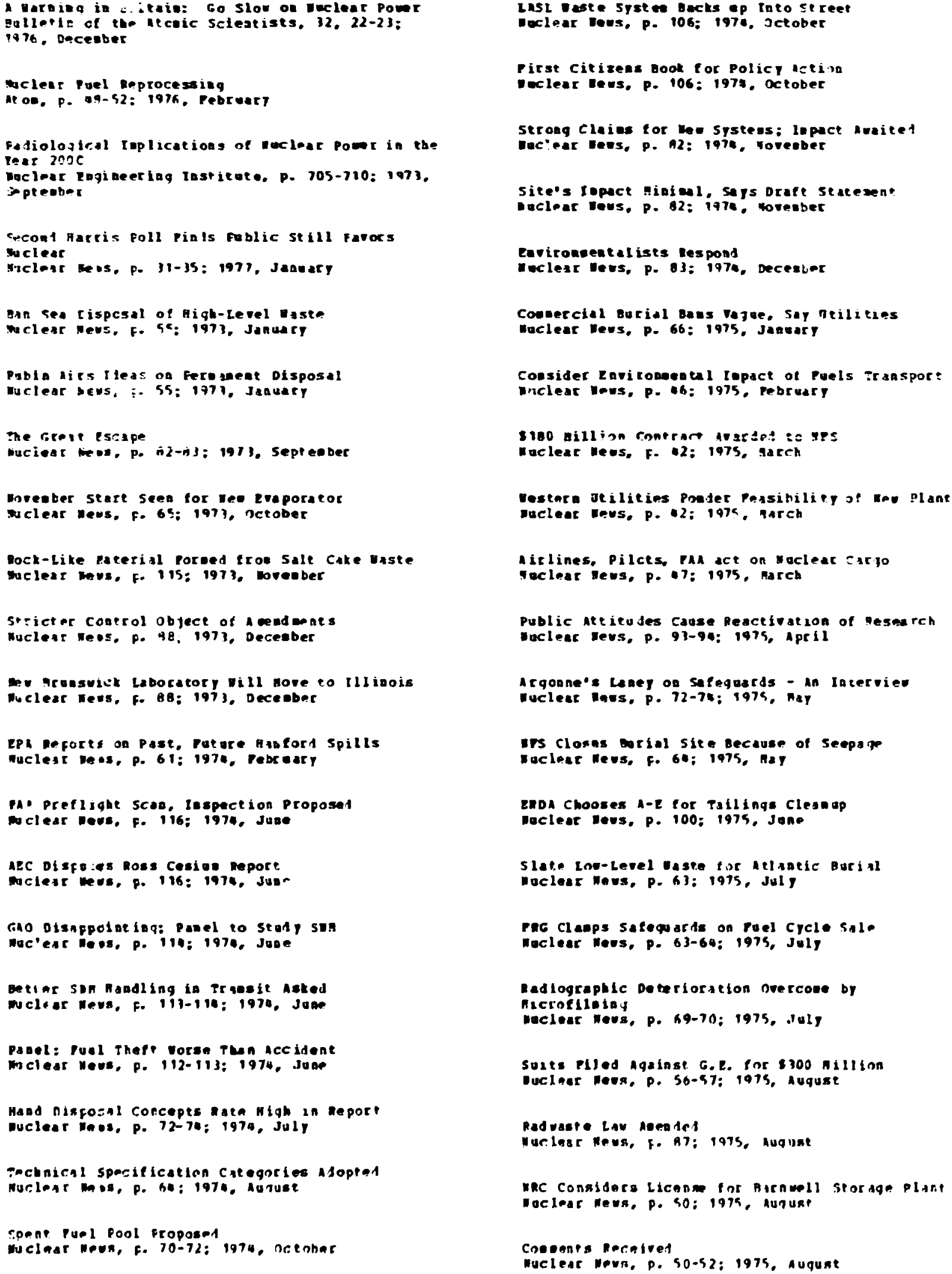




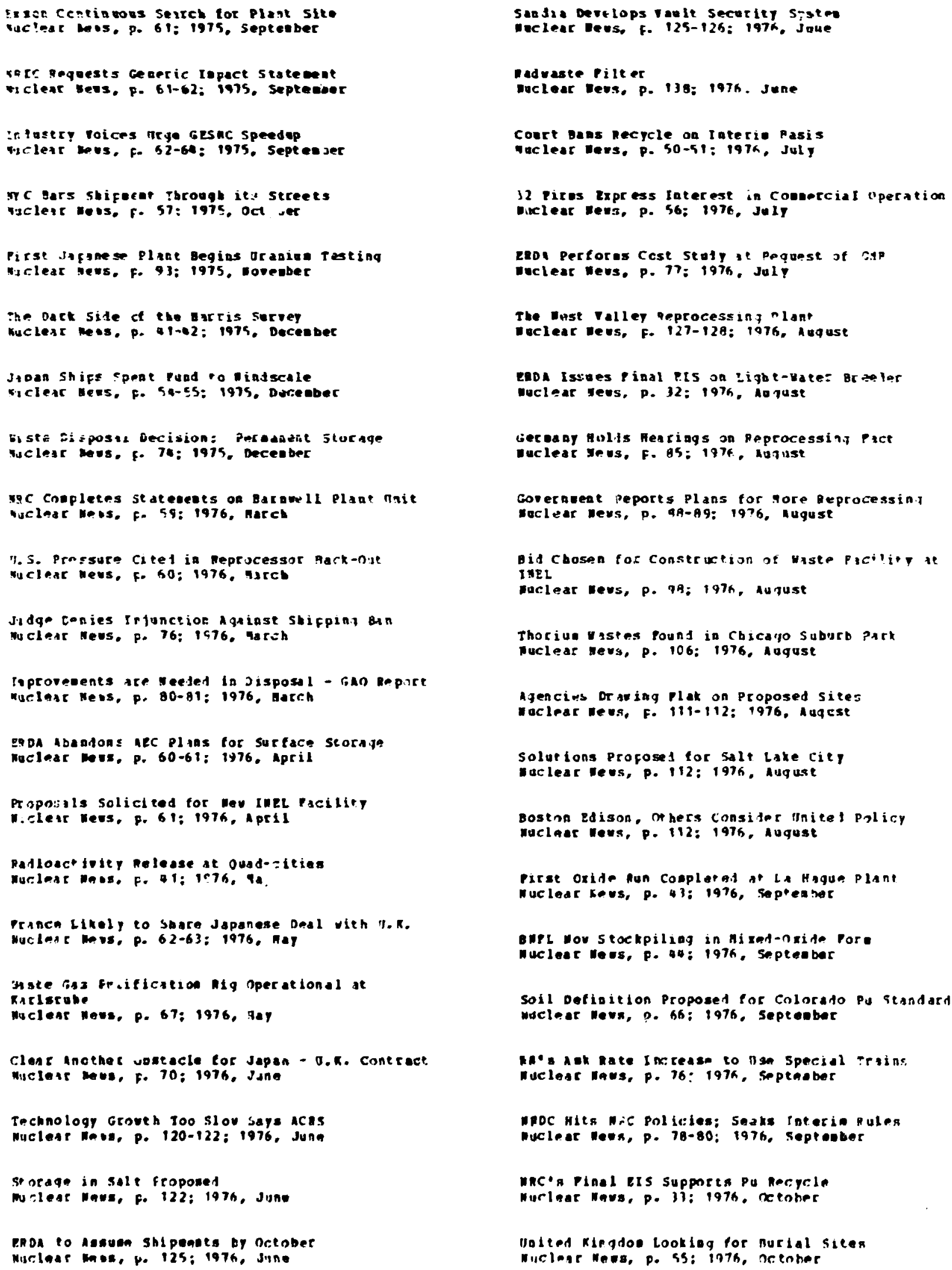

Sadia Develops valt Security syster weclear Beus, f. 125-126: 197k. Jaue

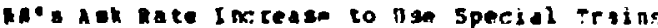
iuclear navs, p. 76: 197א, Sppenaber

WPOC Hits Wric Policies: 5aans interio pules wuclear Nees, P. 78-80; 1976, seppester

macea Pinal ers supporta pu hecycle Murlater waus, p. 11: 1976, netoher

United Rif gdoe tooking for Burial siter Wuclagr Maga, po ssi inth, nethior 


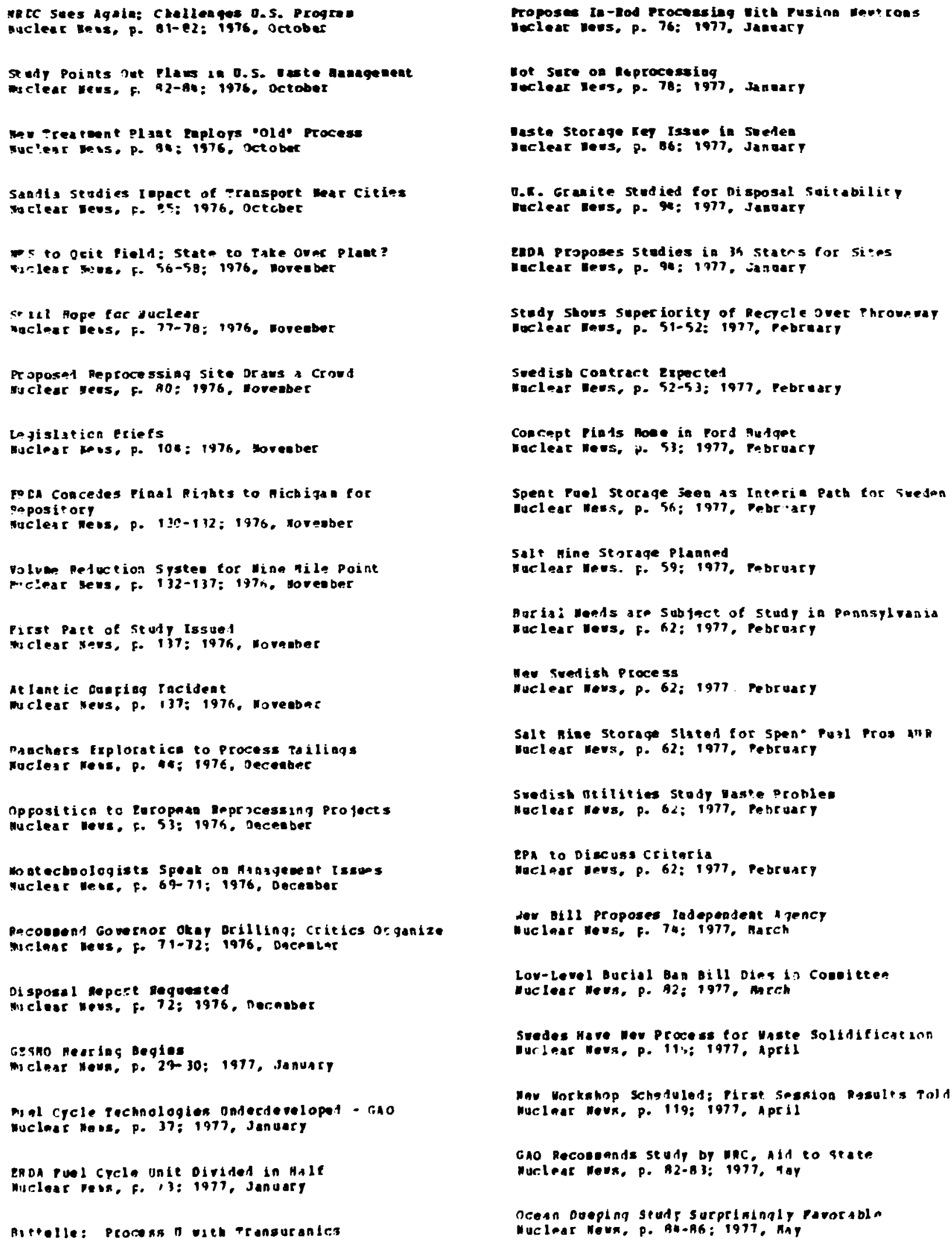




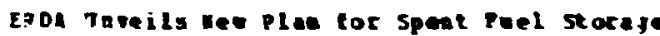
suclear las, p-70, 72: 1977, juae

Ixon to cotione with plaet plats ucleac weos. p. 72-79: 1977, Joes

p-coenendatichs hade on Feal C, le Ioplications meles seos. p. To: 1977, Jane

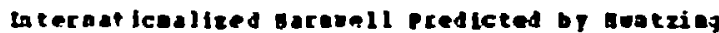
suclear Ieos. P. 7t-79: 1977, Jake

mitile Tait ic provide Pl on Decrele center welear veos. F. I00: 1977. June

ta EA Molds a rectnical haduaste conference vith the putic ic ntel

ricleodics geekly. P. 6: 1976, Jely 15

FDA 5.arting to Pace state Desistance in vaste Do josi:ory Drilling

tacleonics veekly, F. 2; 1976, Juae 17

Courts hqain Say doc Failing to heet ueph Fospoesitilities

sucleonics veekl 8, P. 1-2; 1976, Jolp

ER CA Avaits neter melations it Michigan before Doing nat crilling

mueleovics isekly, p. 2-3: 1970, July is

IF 5 Oult2 tercocessieq, det vaste soliditication rr volee meains

lucleonics Ienkl, p. 1-2; 197k, Septeober 23

A Beal verlt of ooivoidable Naclear

ecoliferation fo pretable veclear Terrcrise

ma cleonics ieek17. p. 11: 1976, septesber 23

2,blockl pladlet heodeent Clears House: Paces conferance plate

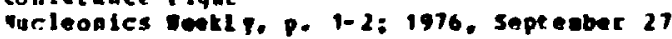

Eltoin C France Ill equally Splie Aeprocessiay ce 3.21n hetric tons

Nuelionfes Iookl s. P. 6: 1976, october 28

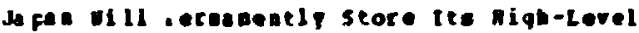
hadose is Gol-gic Porations

ucleonfes veetil. p. 10: 1976, Novenbet it

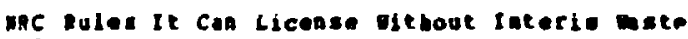
Pule

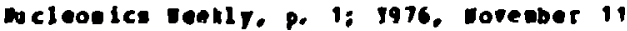

A serite has stoppit prodection of Prance's La Ha que nefrocesn 1 is

nucleosics Jenily. p. 6: 1976, October

The Janifer vas nouthe the lavn

Nucleontes geohl T. p. 10; 197k, Doreeber 11

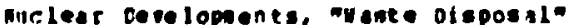

llactical verld, p. S4.: 1971, octooer 19

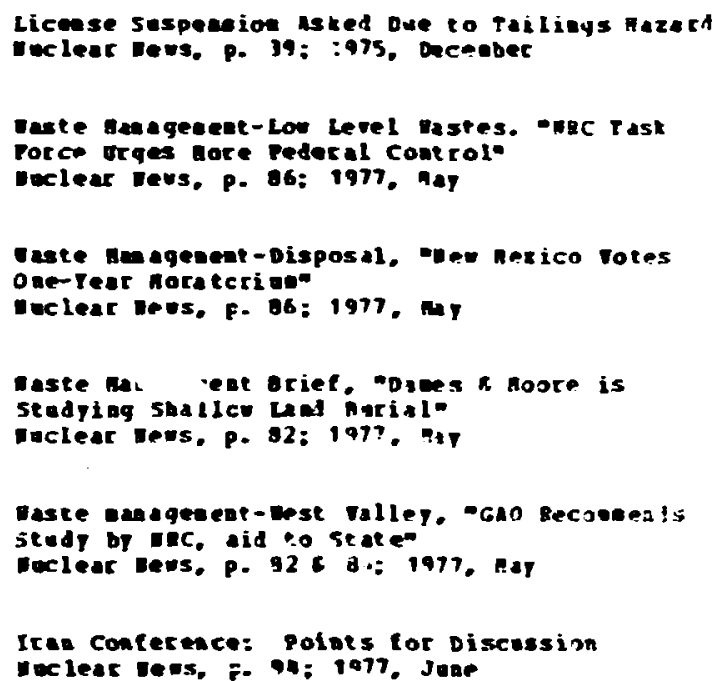

EROA bodget ItI: Cacper Prosras in acpion poclear lews, p. 13, 34, 35: 1777, Jaee

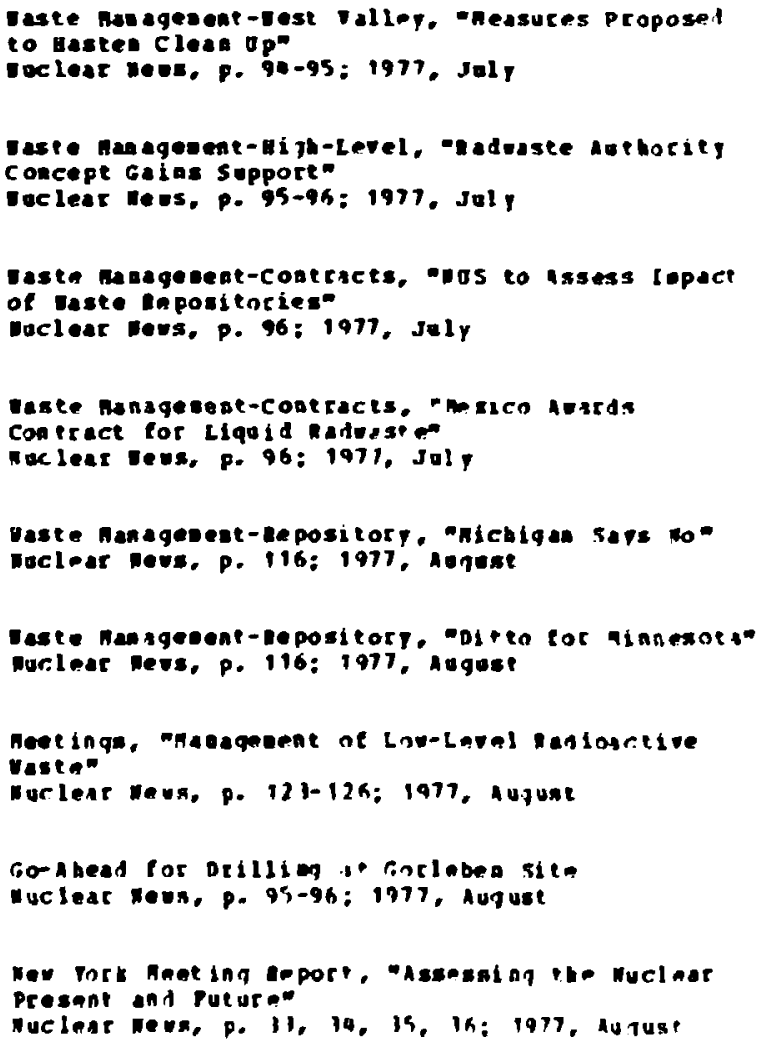




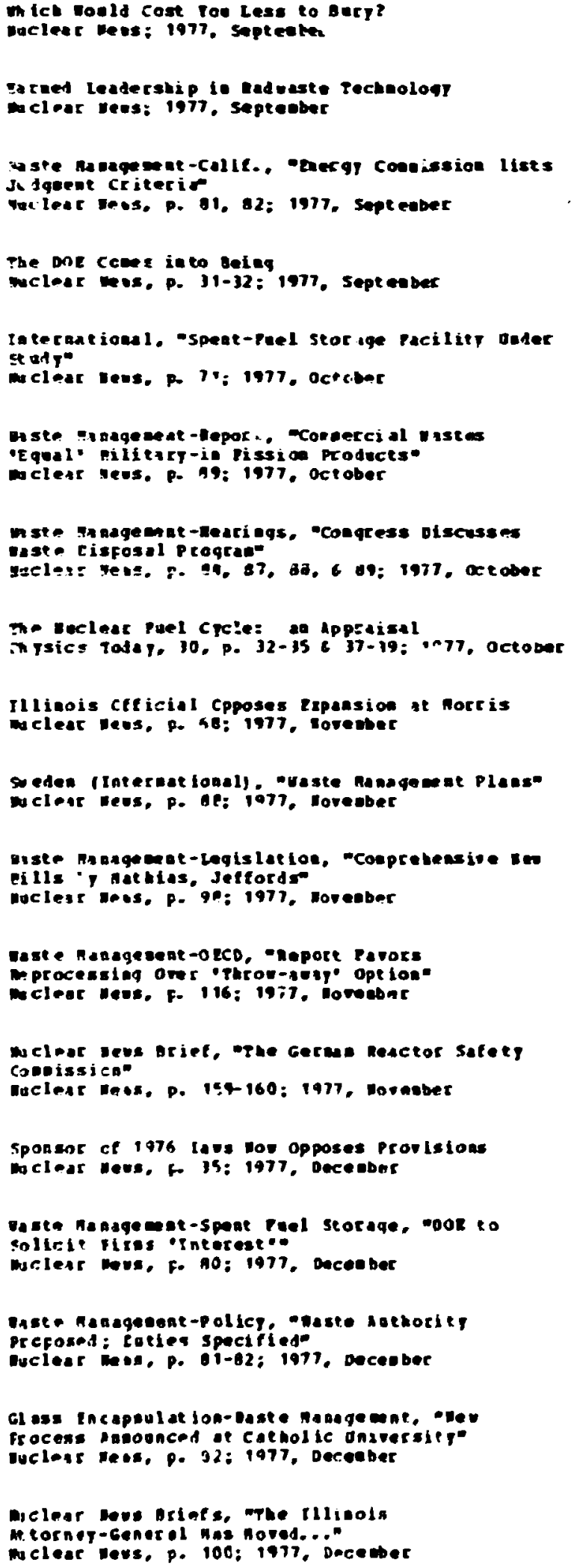

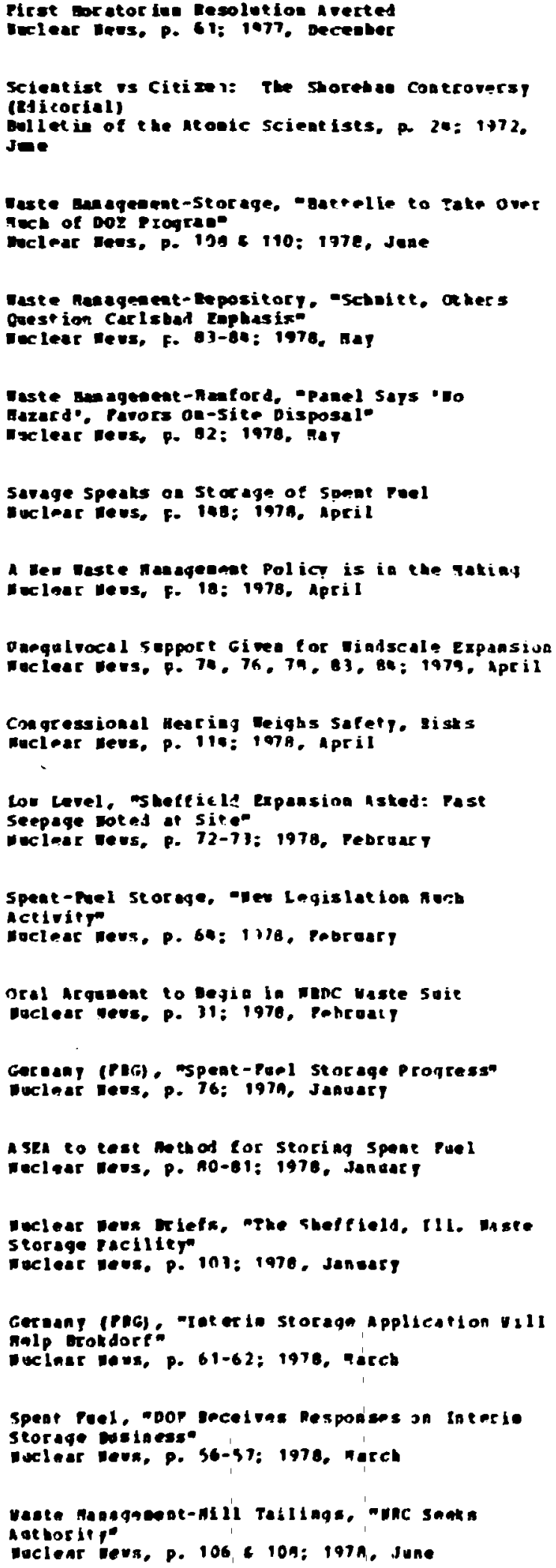


Homets. D.

Matchicq"s Viet

Dulletia of the newic scientses, 32, 24-27: 1976. Deceabet

coners, 0.

viclear fowe

Bulletio of the hooic scientiots. p. 21 .

Eect lon cf radionctive restes incleded); 1978. tares

Pratk, P. A.

Intetantional Convention against melear mete tulletin of the hecolic scienthes. P. 51: 1975. Decesher

Hi 11. 3.

Psiliontive baste hangenet in the onited aingdoe 4t on. P. 250-253: 1976, cetober

Incobs, E.6.

andagene of padicactive vastes - Proytans of the Interadional itceic energy igency

weclear technoloay, p. 300-300: 1974, pereober

Reepia, G.E.

weleat patertal sategands - A professional

Speatis ove

mucleac Do os. p. 76-78: 1974, Septenber

nisq. L.

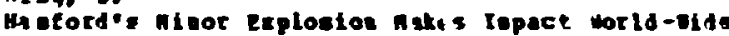
beclear be os, p. 37: 1976, Oct +bex

Krieger, D.A.

Te rrorists ind weclear rechnology

bilet is of the necolc scientiets, 31, 28-29:

1975. June

Woskovshl fo

lecters. piptic zesce missedn cconevet on article wthe public Iesces of tol teprocessing and hodicaceive iasee Disposal-N.N.. Pab. 1977. p. (s-an)

welear leus, po 2h; 1971, Ser

Levenson, n.: zifterero

Te Potlic issues of Puele reproceserieg ond

padioactive vasee of isposel

moclear jeas, p. \$5-18: 1977. Petroary

Le $18 . .5$.

The rialcaceite sale nine

biletif of ethe itcole sciestiece, 27-30: 1971. Ju ne

Lifton, H.J.

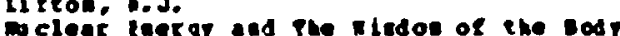

wilet in of the lecuic seleatists. p. 16-20;

1976. Sefterber

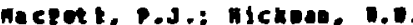

Irst - ibog-Ground eir levable storaqe wethod

for lov-lewel Trangeranic tastes

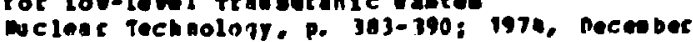

m ve00, C.A.

a ete ol sposal opetose

dletin of the leabic sclentiets, loter: 1977 mireary acclate. 由.C.: Bct, l.t.

ofsposal of radoactive inste it bedded sale Por bat lons

reclear Techeologt. p. 399-408: 197\%, Decenber

AfCxl1. P. P.

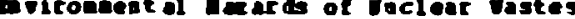

scleace Pallc iffairs, 30, 36-92; 1974, April

Parker. D. H.

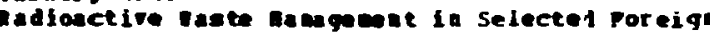
conetries

yeclear Techuolog7. p. 105-308: 197", Decenber

Parae. J.

meorandia on This Iscee espent peel storage:

Seoe old seory"

Iucleet Wevs, f. 31: 1977, october

Piteena, P.r.

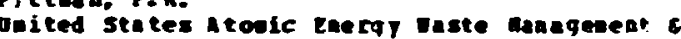

Progians ob foctives

unclear Technolog7. P. 273-295; 1974, Deceaber

neakia, D. L. S 5.G. Deale

letendas of the punlic aboot Fuclear listes

uclear teus, p. 112-117; 1978, Jute

Rippon, 5

Selking a pasis cor Deciaton

leclear wews. P- 50-53; 1977, Janeary

Iippoa, S.

Salzberg Contererce Gets onder may

moclear mens. P. 10-42: 1977, Jene

ILpos, 5.

The recheder Theory

Iuclear Ieus. 9. 94-95: 1977, Logust

aippon, 5.

Prospeces Look Good for Gorleben cencer

yoclear ness, p. 48, 49, 57, 53; 1978, mbraty

loper, P.S.: Dilcos.

Radiological ispeces of éricosenteal tritice

loelear safect, 17(2), 216-223; 1976, Narch-April

Iodan, T. L.

Costherefle constderations of Iuclear Pomar

Inclear Perhool o97, p. 309-313: 1974, Decenber

sapersecio, A.r.

on the veed for a rew ragelsition

Dolletid of the atodic selaneints, 31, 10: 1975, Juee

scheiesen. $l$.

Safegarding Noclear notertals

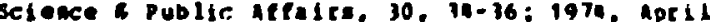

Sheffield

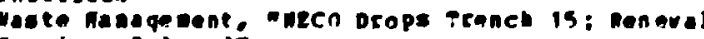

heeringe beleyedr

vaclear Mavin, p. 106; 1970, Jane 
Sheffteld

suclear news. p. 57 : 1970. Hereh

St caf. D.t.

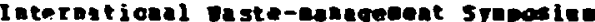

melear setety. 10(0). 502-512: 1977. Jelpagese

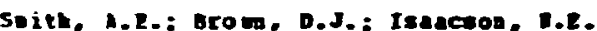

Cheotcal Betrafor aed Growd noverent of selected

padioneclides

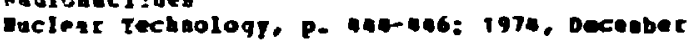

Soeth. J.G. and others

I! utoniug necpcle: The pateral step

science Patic ictars, 30, 18-22: 1974.

to mober

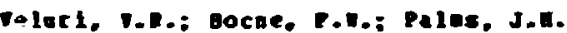

the Raviconencal Iopact of Cesfiu neloasel by a

Declear pel - Reprocessing plane

welear satcty. F- 580-590: 1976 .

septeoker-nctoler

top nippel. P.: Dillians, Dh.

Energy taste and Jeclear pouet Grover

eullet is of the necetc sctertista, 32, 18-21,

aA-56: 1576. Decenter

Ton Mippel. 1.: Gillians, D. B.

en ergy teste is the cont scena rio

Eullet in at the leceic scleatists, 32, 49: 1976,

neceober

tiltich, n.

torrorists reep out!

gulletin of the necoic scieatists, 11, 12-16:

1975, hay

vitte, s.o.

Dationctioity hasagemat

nnclaar pose, p. 65-66: 1977, Jely

Elfa, B.: Latert, H. H.: Molde. G.P.

vill There 0 though PIntosinet

Mucleer ces, p. 72-78; 1977, Apr 11 
25

NUCLEAR INDUSTRY/UTILITY PRESS 


\section{BLANK PAGE}


Vieve Ditfer on Daste Disposal veclear ind astry. P. 25-28: 1976, Wey

What Dif eritle luclear hopore mally say? llectrte Perspective. P. 2-6: 1976, Day

Its Pary, Int Pear

ht onic If lustrial Poren, Iopo: 1975, Jandery

Fublic abivalent bout neclear pouer

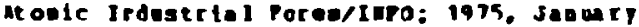

ERDA Fill Gake Weu lase stody

itcole Indestrial poree/1upa; 1979, Apr tI

ypC Propcses Delar ch pecrcle Decision

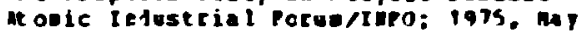

Pa da the Seet Pands for Iase Proposal

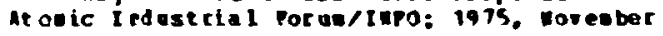

Safe aste Disfosal Peasible, Dec says

atcolic I rocstrial Porde/Iuro: 1976. June

wase blsfesal Urqent to Pubiic. Brock Says

H. onf Induserial Porea/Iupo: 1976. A ugost

Quleating in Radwate Inpaces Held Insufficient

- celc Indestrial lorue/Impo: 1976, Nuyst

UAC Hust Recon: i fer Puel cycle, Court saps

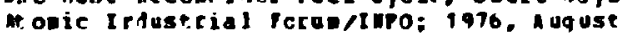

He Grsic study Deleased by arC

lt ooic Irdustrial Porun/Impo: 1976. Septeaber

Waste Ru lesabing to be heopened

lt onic Iodestrial lorus/tupo: 1976. Septeber

Oste Ingaces seall unc study Conclude

ht onfe Indestrial poreo/lupo: 1976, October

Wase macugeceet Prograse serrered

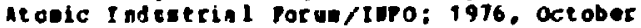

Wer soek to Hale wey Gate Tanks

itcetc Intestidal Porea/Imo; 1976, October

TRDA Ste fs Of Yaste Progras

atode Induetial poran/Iupo; 1976, Decester

Pl veouide Aseaneed In EPar Journal

Atoolc Iedoserial porun/Imo; 1976. Decenber

me veste frcarse oreus sherp sesponse

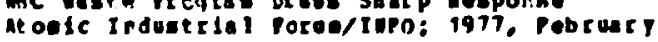

Wiclear shipfieg cauk neets leoorable ob pect

itopic Iofcerlel porou/ropo: 1971. June

We velley site held sate by zea

ar oelc Iedoetrial porue/fmeo: 1977, June
Madoctive vate - probles Child

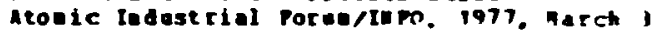

APS seadp confident on vasee wrajecent ntovic Iedustrial porueropo: 1977, June

state Lagislation Conll Ianede Repository Seatcb rooic Iodustial porte/ruro: 1977. June

Bergnan berates moC Docasentary on inse ntoric Industrial Porue 1 mpo: 1977. June

Editorial. What Vill Too Do Mita Tour ustes" electrical vorld, p. 7.: 1973, Septeaber 15

Enclear Developeets, unste di sposal * blecerical vorid, P. 61: 1973. Septeder is

yeclear Develcpents, "Toot varmeran

plectrical vorid. P. 34.: 197k, nay 1

Uoclear Developeents, "Raduaste Disposal" lectrical vorid, P. 29: 1975, na)

Mclear Derelcpents, "Hose handle vastes" electrical vorif. p. 30: 1976. Auqust is

Noclear Developarts, Maste Manageane" plectrical vorid, $p$. 23: 1976, October 15

Wuclear Derelopents, "Daste hanagenert" elecerical lorid. P. ja; 1976. June 15

Maclear Develcpents, Taste njsposal" Electrical worid. P. 34: 1976, woredber is

Declear Developeeats, "Llcensing Actirity" electical Vorid, P. 34; 1976. Voreber is

Der Stedy Doubts A-ilaste Safoty ledestrial pesearch, vol. i1, P. 12 \& 34 : 1976 woven bat

Abrahaseov varas California Hearing of hajor Accident Rist in Poel crele vuclear roel. p. 9; 1977, January

world wes beat-wuclear

Electrical Dorld: 1977, January

Morld Mars Beat eeed ull1 Build Six disposal

Sites tor madionctive Nocleer wasten

electrica 1 orid, P. 13: 1977, January

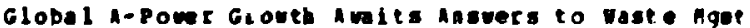
engineeriag Hers Pecord; 1977, June

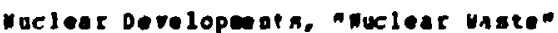

electricsl vorid, P. 26: 1977, october

Odall suggests Incent tres to raln lcceptance of wuelaser iepositorios

The Energy Dally: 1977, october 14, Pellay 
Suclear Cevelopente. "Faste storage"

rlectricsi vorit. P. 32: 1977, October is

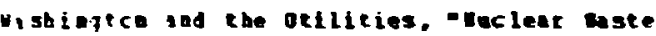
sutile lines bet aq orave *

Public deilities Forteighe17. p. 30-31: 1977 . Jetoter 27

ushingten ad the otilities. -meclear deste Folin Arocaped"

Fubllc nellities porenigtely. P. 30: 1977 . wover 10

Wucloer cevelopents, aduste storage"

rlactricel vorld, p. 32: 1971, Doveaber is

mshingtch oplate, Wa fateiag the unclear progras" Futr. F. 10: 1977. Decenber

mclear tevelopeents, maduaste Reposic ory" flectrical verif. P. 34: 1977, Deceuber 15

seva stater enact heus to tilt easte Disposil atonic Irjcstrial Poranimp; 1977, Decenter

or to enili sta Conercial woclear laste doeps fublic dilities foreaightly. P. 29-30; 1977 . .2s nuary $f$.

Ca liforaie conatssion pessicistic on Deste At onic Irifustigal foree/rupn: 1978, Pebruary

ntention pacus ca lase probleas At coic Injogtrial poros/Inpo: 1978. April

roologic Jaste nepositories Acceptable. OSGS Paper sa ps

it on ic Irdusetcal poraefrapo: 1978, hay

Report on waste Dlsposal 'misrepresents Evidence"

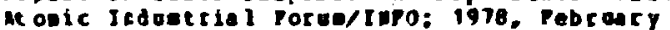

Wuclear heste leport cones troe rask $0::=0$ Public ntill ties portightly. P. 36-37: 1978, Ap 1113

Wi le-iveluation Deadiline for pirst Waste pepositoty

Atoulc Irduserial Porue/Inrn; 197h, hacch

Sodish Utilftea sopply gaste plea

at onic Irduetial Torug/impo; 197k, hareh

covernors set Iaste Becision

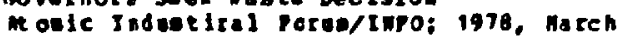

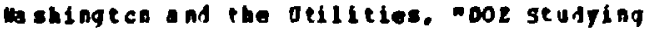

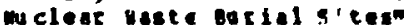

public nellities pertniquely, p. 31: 1978 po bruary 2

In destrial progress: ceraeic cobeiners to Pncaprulate Spant Pual hors tor storage of inclear iaste

public ntilitins portaiqutly, p. 92-53: 197月, in nuary 19
Allee. H.

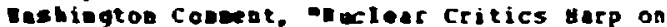
instexs

electrical lorld, p. 25: 1975, Match 1

Anderson. 2.1.

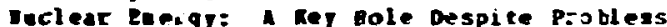

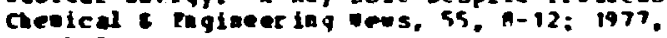
nerch 7

Casbolt. C.

a tepcrt on the peasibility of padionctive taste Bacaling

foblic otilities portaight17. P. 16-18; $1+75$, Jo 3

Cetaraggh H. R.. Jr. (PA.)

Radiactive ouring $0 . x$.

plectrical vorla, p. 71.: 1973, October I

Caraparat, h. A. Jr. J

the hanagesest ieport, -Till the lod of miga"

rectrical vorld, p. in: Po78, January is

Colen, B.L.

Sowe issues id the uncleer powet concromesy poblic otilleles Porenighely. p. 11-34; 1976. Auguse 12

Cosar, C.L

Elological pisk, electicity production Palo alto, CA: electrir pover Resecech Institule $(25-2):$ 1975, Doverber

Conar. C. L .

Pueting Pistontos in Perspectire

electriral vorld, P. 39-41: 1976, Deceober 1

Cranford 0 .

The Critical Issues: An Indusery Perspective lectctc Parspectives, p. 12-16: 1976. Jandary

Deu, G.J.: Williaes, R.P.

stetus of Consercial wuclear High-Level aste Disposal

Palonito, CA: Electric Power Desearrh

Iastitute. Np-44-SE: 1976, Septenber

Dau, G.J.: Vllizas, R.P.

Secure storage of Radioactive unate

Aware. P. 6-11 (Reprinted fros EPaI Journal.

1976, Jul Y/an qust) : 1976, Oct oher

Dullien, R.C.

woclear-vaste Problens are opaed Lase

Technologlcal than Iest teutional

Energy pesearch iaports, P. 5-9; 1. h, Dacenber

Exloni, 5 .

Meneved Confidence In wuclear pover

Auere Nagasine, 86, P. 2-6: 1977, Noventher

Parthing, J.r. (E. R.

Dadvaste an -ilodge: Dlaposal is Difficule

elecerics 1 worlA, P. 40-43.: 1971, Decenber 19

Farthing, J.G. (PA.): Pletean, P.K.

on the ousticn of mad ascetre usses

riectrical vorld, p. u4-46.: 1973, detober. 
matere. J.

Miflotonte nazerd masere for entiol solth

pidoact tre dase

nucloex Tochnology, p. 180-189: 1975, Earch-April

Hill. 1.

the legal side of the necleet satery arqueset

pulic arilitios perteiget17. p. 11-12; 1976.

Jusert is

Qx 11. 1.

a Intersiov ith atr president carl walshe oe

suclear feet gr o the carter adeleistratioe

fublic neilf ties partelquely. p. 38-bi: 1977.

aril io

Hi 12. 1. L.

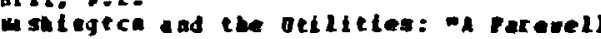

getervifo wit we chairoae Douden

pablic ntilities portalquely. P. 24-28; 1977.

Jaly 1

moer. C.

The hesecy of vuclear bisent

electric ferepectives, p. 20-23: 1976, nap

In ler, 1.

Indusery Devel openacs, -observers ob ject to

D- Dasie seod $7^{\circ}$

llecertcal ucrid, F. 26-27; 1976, Dec. I

Ioler. I.

Iafustry oevelopents. esuedes hay store voclear

waste in Rocko

Electrical Gorld, p. 22-23; 1978, Jenuacy is

IV lev. T. (Ed.)

wev pales on uclear-vate transportation

rtectrical vorld, P. 24-26.: 1973, hay

none 1, 0.3.

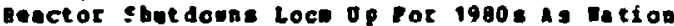

hoce ceeper laeo spent fael pickle

electric Perspoctives. P. 13-17: 1977, mat

Saleh, 0.5.: Lancester, M. A.

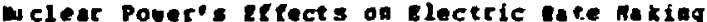

puble deliteles Perestgtel7. p. 16-22: 1978.

pebroary 2 
ENVIRONMENTAL PRESS 
ED DA uste disposal

a iticel ness, p. 11: 1976, Aegest

eavironental oriets

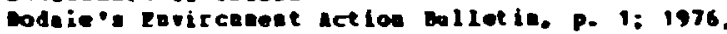
$\operatorname{sol} 12^{\circ}$

The hazarts of Ionizing tadiction

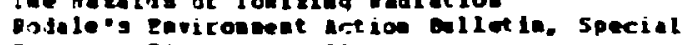
Is see: 1976. October 10

Eetecty Seets to avoln bur tes

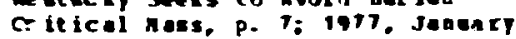

Laceetes* Iaraing

Critfeal aass. p. T: 1975, April

Wuclear fowt: A tegrouplag os Detreat?

pesces. P. 6-A: 1977, Jaberp-Aarch

Duclear wases: the second Tile Aroend

favircaefetil Actice. F. 10-15: 1976. Jome 19

Flutouto to Cane 116.000 caecers

cltical nass, P. 1: 1975, Aojose

Radiact ive Leaks

Crittcal anss, P. E; 1975, April

moprosaing plant sabs License for vaste seorage cr lejeal hass. p. B: 1975, hequst

Soviet vate peplosich lleqed

Q tejcal hass, p. 7: 1977. Javury

St otes are Prepared to lage har Agelast

cadoective inere bleposal plab

Critical hase. P. 9: 1977, Ape il

Wuclear Garbage: It Just Don't fo hat

tot ha Apert, p. 10-11, The meclear bioedorn:

1976. Angeet

I* sab Prancisco bat sate trop plutoniger lot has ipart, p. 10-11, The Meclear oloodoen: 1976. Ne quet

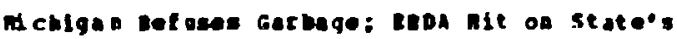
11 que:

be hon ipare, p. 10-11, The Daclear blowdoug: 1976. Aagest

Inforectica nood nese Transporetion

virosectel Actlon, p. H-4: 197k, Decenber 25

Meilrouds Peir Daste, Po

m vironeentel ncelce, 3-0; 1976, Deceubar 25

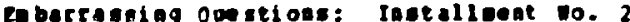
peo, Sal iradedeco, III (2), p. 3: 1973, noveubar

Wn wee Dieposel in S. Caroline nisted

popole 5 pover, p. 1: 1970, Hay 17

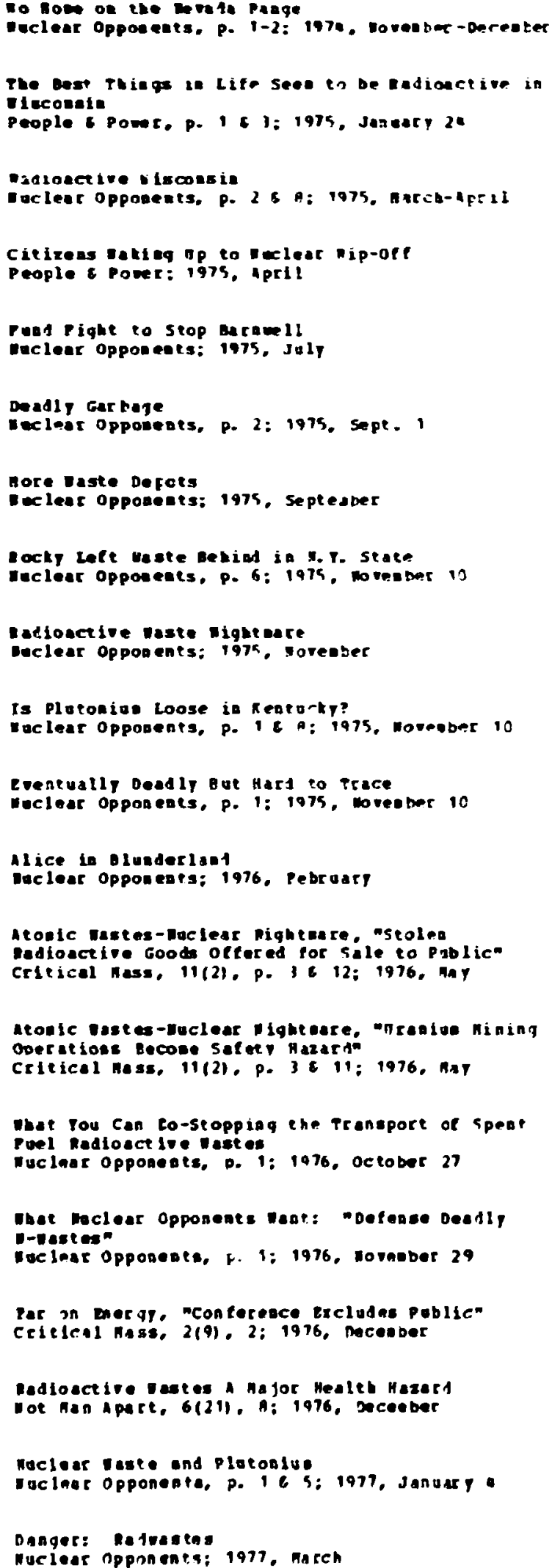


E.l Daste Trinsport marards Goo welear iffouents. F. h: 1977. Mareh 10

pianesot. Ges yuclear waste Storage nesa w clear CFponetes. F. 5; 1977. Aet 31

Hexosain wolves to gan wase dop w clear cpponetes. F. 5: 1977, day J1

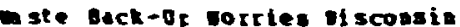

we clear rffodeat5. Fo 8: 1977. Wey 31

W- Mrico Paces Daste

is clear ofponents. F. 2: 1977. Hay 11

Canaba's baste noes

miclear cffoneats. Fo 3: 1977, may 31

Onbi:- noci act ladioactive laste

citicel hoss, $3(0), 3$ : 1971, Jalt

Mirhigan vaste matcher

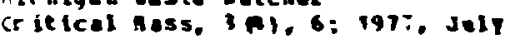

Bebind the Daste stee search

meleac Cpponete. F. 2: 1977, Jely

Whiggeca Poat Coluedist Gerge P. Dill cecenty re portert:

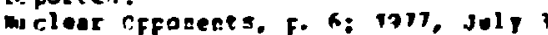

ur ge special Conaision on uelen uses

w clear crfodete: 1977, Auquse

Work state iase Opset

ucleac Cpponeres, F. 1: 1977, Augest

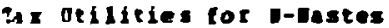

mu clear cpponenes, fo i: 1977, Auguet 1

- estes is Sevage

Mu clear cpponente, p. a: 1977, aguse

Date hat scorcter

bt hes apere. p. 15: 1977, Dacenber

The Dase wo One Wance

the has ipare; 1977, Decenbar

wo Nore veste, Plasds Illisols

the hat Afare: 1977. Deceober

melear wase a ceatly lequef for our cbildren

nuclest Cppomats. p. 1 t $4:$ 1977, Decenber

Protent vaste iraneportation

nuclear Opponente. p. 1 6; 1977, Decenber

Lat ve the Orantue where le lias

Muclear Cfforenea; 1977, Decenber 16

welear forer: inceogy of a Palled Techaologp

Muclear npponente: 1977. neceabar is

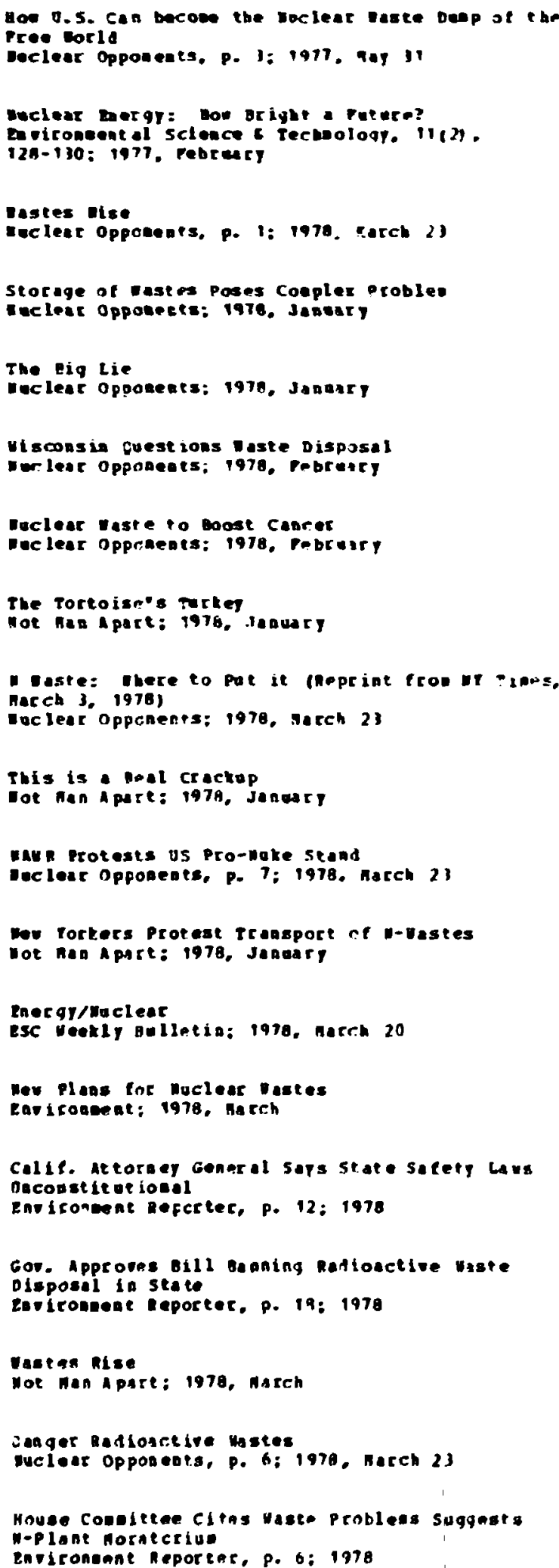


te seloe Taillige lecoral

nigh Conery peos, 10 (7), 10: 1970. Apeil?

The sierre club

w clear foeter and the sterre Cleb

esa Prancisco, CA, 12 F. paoplet: 1977, Bt

Morateeseca, D.

werg Pcifey ift Gen

boironent, 16, 2-3: ig7a

Abrahassoa, C.E.

meleac thefe s inclear parks

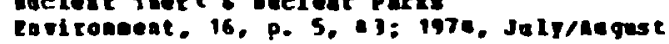

Abrabaneas. L. E.

pouerd Gartisoe seate

enterobeat, i7, 3-5: 1975. Junuary Pobreart

aren, a.

ct $x$ tenes

science and Fubl tc Mffairs. printed in Weclest Opponenes. C. 8. Aerch-April 1970: f. 3: 1970. Jan.

Auqustiae, $\mathrm{B}$ -

Indestry ibancar yastes and lesponsiblities

cr leicel hose, p. 5, 7: 1977, Jaovert

lugostiae. 8 .

Padicactiv Daste scranble

Cr Ieteal Mase. P. 5: 1977, Pebreary

Barstis. J.

the ist hines. Mills $\&$ Dorsbips padioactive

No el-nraoien

High Coater wevs, 10(5), 1-8; 1978, Murch 10

selser, T. n.

sadiapper porus: Is soutb carolien to becom

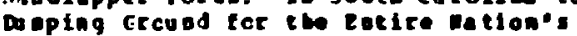

Daslouctive Dastes?

sa ad la fper, F. e5-52; 1911, May-June

Derger, J. (Ed.)

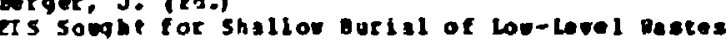
nop han ipart. p. B-9. The iuclear dowdowa: 1975, October

enrger, J. (ed.)

Tee people Ccrevel aeted is Matord Explosion

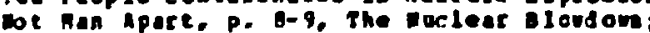
1976. OC Paber

Erqer, J. (Rd.)

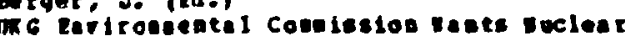

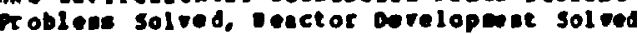
lot hes Apart, p- b-9, the Ducleer Slovdown; 1976. Oefober

Ba rqer, J. (28,

Gareas Beactors choke on Dastes

to nas ipere. p. 12-13, The toclear bloedoun:

1977. Herch

enger, J. (2A.)

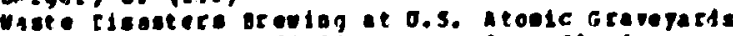

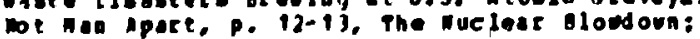

1977 ing

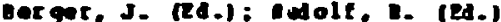

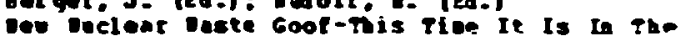
nodeon.

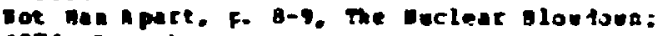

1976, Deceder

Berger, J. (2d.): Dadolf. P. (Ed-)

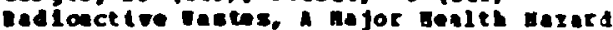

Dor hav part. F. E-9. De nucleer blostoon:

1976. Decebot

Ber gat. J. (ed.): Indolf. P. (ed.)

prevel ceer of Fiport proqrae

bot has apare. P- a-9, The ruclear bloutoun:

1976. Decesber

Barger, J. (Ed-): Dudolf R. (ed.)

Experts olscess inelear Gurbage

bot han apare. P. 8-9, The waclear bloudowe:

1976. Deceser

Cor. 3.

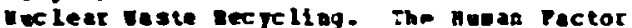

Spreads cediation to Cítizens mer dispostl sita

eavironsental nct toa onlletin, pe 2: 1976. Tay 29

Cullicore, D.

Madtouctive Deste Boried by the Bereacracy

Eaviromeatil Action. p. 1-5: 1976. Auquse $2 \mathrm{~A}$

Drake, G.A.

The Case Agilase buclear power, Daste Disposal

Leadership fl stutemat bofore the covernor"s

TAEt porce on fuclear masee di sposal

unclear opponenes. p. 7: 1977, harel 30

Edsall, J.T.

haserds of wuclear Fisstor pouer o the cholce of

Ale eranteres

Envirousetil conservetion, p. 21-30; 197e, spring

Rdsa11. J.T.

Pareher Coevents on hazards of Docleer pover and

The cholce of alterative

zarizonesatal Conservelion, p. 205-212: 1973, inesen

Est. 1.

Eadiatias expcsute videspread: Cancer pate Migh crielcal mass. p. 2: 1976, mat

Porab, J.: Leolne, 0 .

Mnclear icste lusp: oninous proposition

pecleer opposents, p. H: 1977. Decenber

Pord, D.P.: Tendall, H.D.

oclear misintoration

zovicoesest, P. 17-20, 29-27: 1919, Julp/Auguer

Garnet. P.

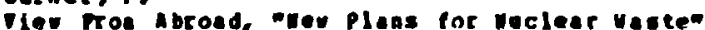

Eavironeene. $20(2)$, 4-5; 1977, Augase

Haprood, 3.: her, K.: Mesnikoff, He: Schlenger. B.: Tases, P.

The cose of tornisa le oes

Environcent. F. 17-20,25-26; 1976. Deces hat 
lititer. 5.

Di liat loe iffect: ouderestiented

crit ical anss. p. 1. 12: 1976. Cet

interes. 5 .

Cilizen's Gatde io meclear rover

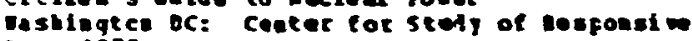
La: 197?

L ppe. F : Bowe R.

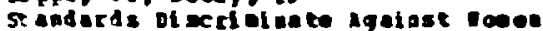

celtical nass. P. 2, 11, 14: 1976. Wa

movick, S.

5 reatece pillton rears

moironbert, P. 42 7 : 1971, wored ber

sovick. S.

pepere card on weclear tower

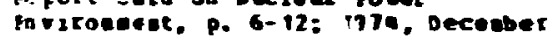

Movick, S.

Wuclear Preeters

Fnvizonerat. P. 6- is: 1970, July/hejuse

frenlte. A.

Gandi: weclest iste

favitonest, 19, 2-1: 1977, Aogust

pictersen. ..C.

sprack for flotonies: Mritish meclear Puels

- I initef*

Envircecent, 19, 41-4; 1977, Erch

pollock. D.

If ca to survep acst states for daste site

o itical sass. P. ;-t: 1977, Janery

pollock, 1.

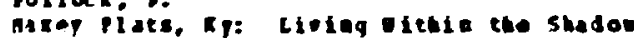

trical nass, p. 6; 1977, April

polloct.

unc iriticized fc: Abrlibe hare rdoes cargo

mquilaticas

critical Tass, p. 7: 1976, luquat

mollork. P.

in poifilcal pallowe of haxep pliss

ciebal gass, p. H-9: 1977, Aprit

Prpte, P.P.i Prgde, L.T.

soviet meleer pover

envirogecat, p. 26-30: 1974, Aprt1

m Des. 7.

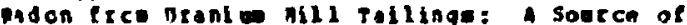

sign I ficat rediat lon mazert?

enof roncentai neneqeesine, I(2), 119-14s

estitoft, $n$.

mpensive inetcheane

pari roneone, 17, 28- 35: 1975, Julp/August

menehall, 1

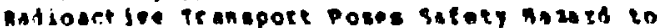
putise

Criefeal 4as, D. 12; 1977, Apr bl zasentix.

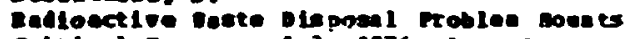

crtelcal mase, P. 1-2: 1976, Mequst

swer. E.

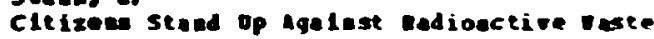

sil peeats.

Evironecel act 108, p. 2-3; 1976, Decmber 25

swaen, a.

Dor to Till tear Prieats

Enviconectal Action. P. J-e: 1976. Deceber Is

swant. $\mathrm{L}$.

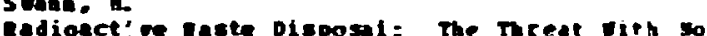
solvet on

codale's Entroment action Bulletio, p. I-9

special rssue: 1971, wacce s 
LOCAL PRESS 



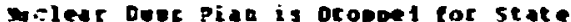

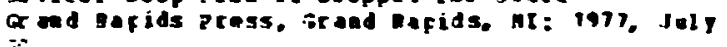

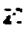

Dichigen TCl1 te's we ielet we as site for putere inclear poef

ae jerccit News. Detcoit, TI; 1977, July 21

sifety ccess pirst ta berial of suclear lasto opprifre ter al panal

the betcclt sevs. Detrolt, at: 1977. JelT 21

ai chipar coult neccee oi sposal site

becalt. milliagtea. ar: 1977, July's

Aills Gerid ean G-Deess Bere

-he crove boflds press: 1977. Jane 29

- Doep dealing Criticitel

The cetccit pree ?rens, Detroit, ar: 1977, J ane 15

welear wase surial wot for us = nilliten

nidiend vees, Midleed, al: 1977. Jabe 17

Senate gules od weclear Disposal site

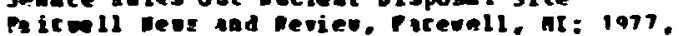
$\operatorname{sil} 7$

niliken wefects state

The Detcoit Eevs, Detraie, nI: 1977, Jom is

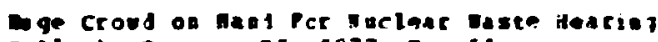

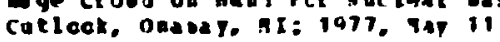

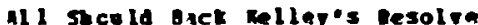

port necce times merald, poct horon, ar : 1977. $\operatorname{nat} 4$

Dobes on melear iaste plap

morae Meus. Rearoe, HI: 1977, April 25

welear roplag in nichiga sepeied

pouville Mecald, Feantille, it: 1971, June 10

Chece of weclear beep Aleost zaro"

arlased Tribare. Aelonis. Al: 1977 , June 23

P se selil destified on malear varter

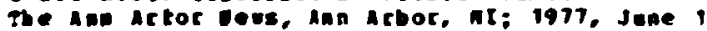

ot cerbucher flas basolution draing wuclear

Clsposel vetc

Al pene lees. Alpoes, in: 1977, nay

A-vance ruspieg Pollcy Critlcized

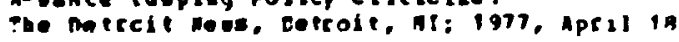

se ore would peve veto of moclat buepian

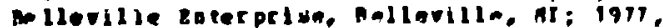
Aprit 1

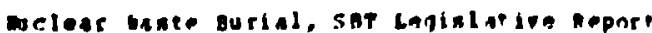
ioples

Al pona weos. Alpast, ir: 1971, April in

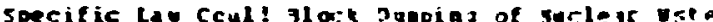
oallorille onterortse. milovillo. ni: 197 nor il

Relley Destlies oup ob fections

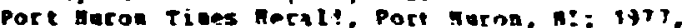
Mar eh 20

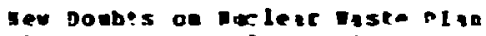

The stare Jceinal, Lastag. AI: 1927, Ixil

Dr. Orake offers Alterati ves to fichig un is

$x \rightarrow$ iste sito

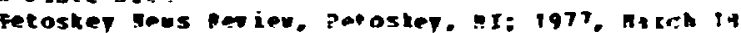

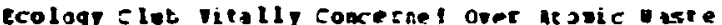
Dueping

migers ciet advace. Popers ciry. HI: 1977. March 17

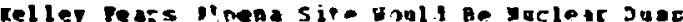
por the voole verld

phe frand popids prass: 1977, Haceh 16

Doulf Probibit hucleac laste Duep In st tee Par wall vers and Revim. Farmell, at: 1977 . Pabreat 17

Wuelear Pane I Draus 100 to Sif" Task Porce geport abelene mich. Heus: 1977. Eebraty is

Millike trucker Plis lins sopport

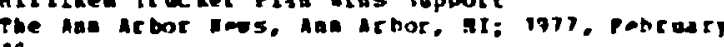
10

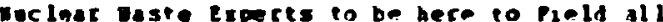
Otestinas

alpead. Alchiqa vers: 1977. Pebruary a

Dill veald Give state a To on s-Daste Cuep

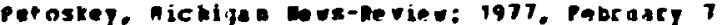

veto nique veeded in vaste peed The stafe Journal. Laesing. Gichigan; 1977 . February 7

Serjet Controls for wolent Doepind Asted alpena. Michigao Weve: 1977. March?

Dill unuld five michigan Feto Porera

The sagina wess, Sagione. Ficbigan: 1977 . Pebrust 2

Rep. Stevens trpian to piahe vicleac oisponl sile seraitsland resocter, Indi on miver, Airhigan: 1977. Januect 27

Lis ison coesteree orqanizh a

ilpens, Hiebigan mevs: 1977, Januart 21

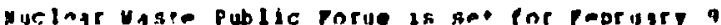

lipeni, Fichi qan weus: 1977, Jinuary 21

Murlear Uanta Dusping Pea?

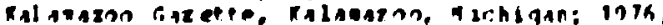

cal tober 12 
Step's Atenie Dep

the Grad lafide prese, Grand apids, Michigas: 1976. Cecenter to

Conoisstowers pase Besoletion Opposing Duclear Di sposal site

Indian Pier mescreer, Isdian Miver, glemigen: 1976 . Jel, 29

LPD 6005 Det1-Liter

wos, Segiese. Hickigae: 1976, Jel, is

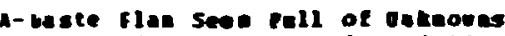

The Detrcit vees, Detroit, ijchigan; 1976, Jal 7

Welear Teisens Ret be Deried onder vichighe

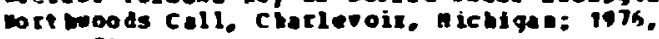
narch io

Senate Adefes Legislation oe wate Defosit

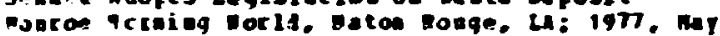
m

vaste Perales O111 adopted

shreveport Tibes, Datod Douge, LA: 1977, hy 20

Muepleq till on'd by onit

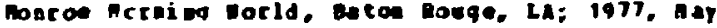
19

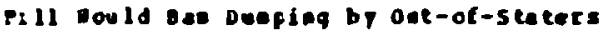

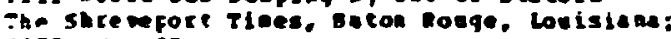
1977. P1Y 19

manlag selll onsere of Unke oue pate

mieden fress-heralj, hindea: 1977, May 19

Shrevefcret vould oret nelos Carbide "Bubble" Tiden Press-fiereld, $12(28)$, Tiedes: 1977, ngust

wa qgoenet cesponde to letter

ni onea Press-herald, 12(22), Misdes: 1977, Aoqust 1

Thaber lases cosg

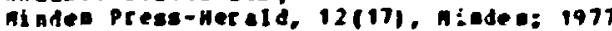

Cans tc cobat noke poblet

ni adea press-becald, Minden: 1977, Julp in

Dow -progaratlos" Aretery

nindon Prees-nerold, $12(11)$, ninder: 1977, J el is

Teds III hate Duke beclsion

ni mes Precs-merald, Eladen: 1977, Joly 19

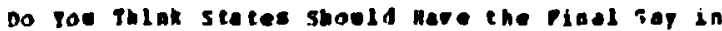

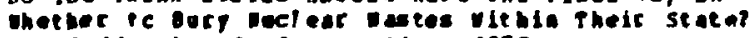
soundoff, rhuredap's oesetios: 1977

m wuelect storane set at hect islased menten ifvorita, neton nouje, LA: 1977, July

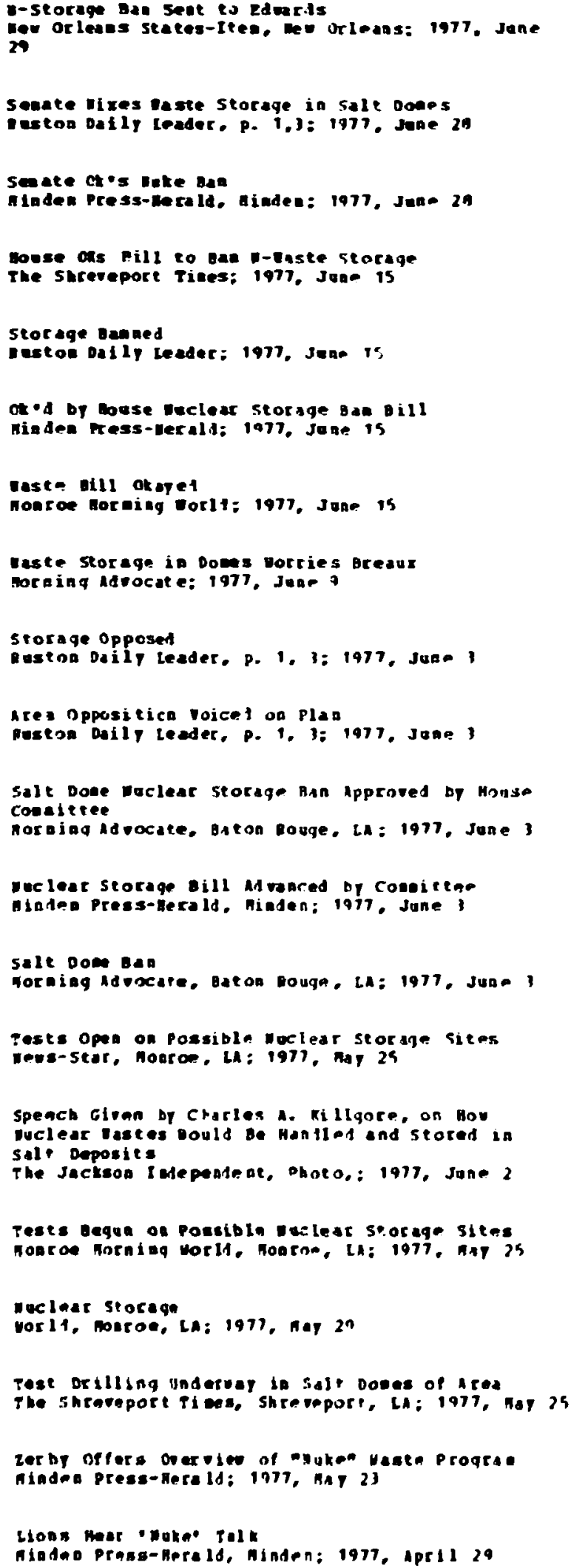


searchleg tor sefe oreporel of nedionction

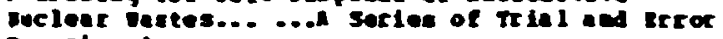
Experienee.

nided Press-nereld; 1977, april is

Citizese haidert madoective storsge (CAns) the bientile Decoctat. Hisdes, LA: 1977. April g

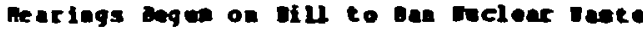

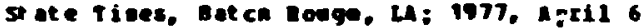

Talt set on clapcsal of ichear astes the fashingter Pose, Iashiajton, Ge; 1977, April 10

Masidears Aicpt besoletion Against melear iaste pieville Ceocret, Arcadia: 1977. Mpril ie

welear Rear tag steged for o obberly mesidents hinder PI so-nerald, Mindev, LA: 1977, April 13

Arcadia Group Ells Eclear vaste Issue

The shreverore rines, Arcadia: 1977, April 13

Mblic Heet:nq held on Issee

tome heraing Icrid; 1977 . April 12

welear sucrige frotented

maton Colly Leader, Auston, LA 1977, April 12

MAP Hears wuclear Talk

Ttaden Press-her old: 1971, April it

Arcadia Posts wecleer taarín

ninder Press-herrald: 1977, Mprll 12

wuclear folicy is at

waroe meroing vald: 1977, April 9

Wuclear waste neet set

nowros ncraing For 1d: 1977, April9

D-naste storage nill nit

streveport Tiees; 1977, Aprtls

Solar tenergy stedied

Shreveport rieas: 1977, April

beologr Preaks?

bo orleans sectes-rter. Dev orleass, LA: 1977, Aptil.

Soll weer N-plane "Poteetally Maxardous"

the strevepte Tlees, Colden, co; 1977, April 7

Storeqe tan sought on Noclear unete

Pustoe Dally Leater: 1977. April 7

Muras wary of state seoriad Wuclear yase

Horning Mifocate, Baton Houge, LA; 1977, Nerch 11

melear cisposel sites erokes meqe tive nesponce Minden Press-heroli; 1977. Marcb 21
La. Legiolatere oill Coasider melar oste

Fress-Bercld, Minden, LA: 1977, Garel 16

Police Jury aenbers ot Conveation

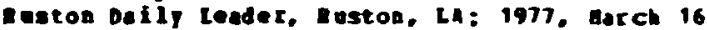

Pol ice Jury-icsociation passes pesoluticas on Tar, Salt D caes

Horalug idrocate, aaton pouge, LA: 1977, Warch 15

Doard refuses to Give salt Dose Testing nat

niaden-press derald, Minden: 1977. Map i7

lgainst ruke storage, Jeteing. Chabber Alopts - Stande.

misder press herald, minden: 1977, Mat 16

Edvards Jol as Porces here to Oppose "Wake"

Disposal

Mindea-press Barald, Tinden: 1971, hat 11

No La. W-Duge, edvards Tous

Wev or ledas states-Iten, Wey Orleass, LA: 1977,

mat 11

Governbent Looking to Dunp Noclear lastes in Salt Doves

Fonroe, La DocLA: 1977, Hey

Sol on Cpposes $D$-iste storage the shreveport Jourasi. Shrevepor". LA: 1977. Hay

Gibsland Residents Right Muclear Waste Pact Dientille Democrat, Arcadia, Lh: 1977, March 19

Peclear waste storage Moujd Crate Bieoville

Jobs, Offlcials pepore

Shrevepore Il wes, Shreveport, LA; 1977, April 29

Deciston on waclear site wot par off

the ofenotile Deacerat: 1977, Mat 5

zAward's Datoo

Wast Carroll Gazete, Oak Grove, LA; 1971, warch 31

Udelear Idste is henting Topic

The Shrevepore II ess, Shroverpot, 2A; 1971, Apr il 27

Muclear vaste Cleposul heeting thls ged.

bienville oneocret. P9. 1; 1977, April 29

huckaby Opposes muclear Disposal Here

bientile beecrat: 1977, Apríl 2A

Wuclas Deste heartigg held

Moncos noraing World: 1977, April 28

Huck aby Opposes Vuclear Vante sporage in state Aadisob Journti. Talludab. LA: 1977, April 2A

Sase Sonq: Duclear experes qo Howe

hinden Presa-Herald, Hinden, t.4; 1977, April 28 
Huckaby Cpgoses storieq belear wasee in sece Tides Sigal, Jese, Li: 1977. April 27

Local citizens nraed to Pighe lgalast warby st crage ef iadioactio uaste press wers Jowral. Sprieqhill, LA: 1977, April 27

W-vaste rispesal hit by Solon the surevefore tives, surevepore, LA: 1977, April 27

welear waste nectiog todat

nource ncreieq verld: 1977. April 27

Duclear gadiation is Deadly, Jeterass angered by Treatuent

Qiest111. Denocrat: 1977, April 27

Reting ct conceraed citizeas set

pientile ceocrot: 1977. April 27

yuclear peet set, storage of voclear vaste

naterial is Issuc

Buston Daily Leater, Duscon, LA; 1977, April 27

Huckaby Againse A-sices

hontoe mereing oorld: 1977, April 26

Springhill Jaycees hear Manaing on storaqe

rinded Press-Rerald: 1977, April 26

welear series ceneiwes

hidea press-8ers14, ninden; 1977, April 21

Pablic neet Set on Nuclear aste

The lostes Dailt Lader: 1977, Aptil 20

melear mate wearing called by energr officials mi aden Herald-press; 1977. Aptil in

Eleards Pespcads to soletion

hider Hersid-Press: 1977, April in

Eacery haswer is coul

nonroe neruing Oerif; 1917, April 15

Huckeb : Energy Preseing Issue

mintes press-Rerald, Hiedes: 1977, April is

A-wate coery raised

no orleass states-Iten, Dev orleans, 6A: 1977, As ret 11

w clear Dase secrage to depand oo bencties

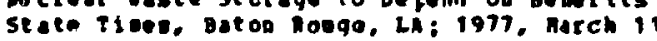

Sa If Dene storage: Apecial mepore, Part 1 : The Argusents for crode cil

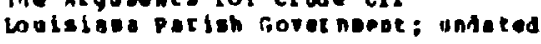

se orage of Muclear Wases in ha. Byed

the Datip Record. Wen orleans, in: 1977, Janwery

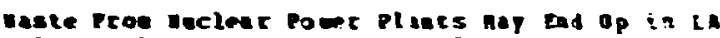

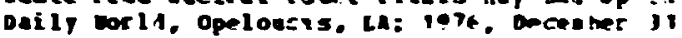

Declear ieste sites contirdet

post-sigal, Crevlet, LA: MTh, Mecaber it

La. Salt Does Wh thons guclear Deste

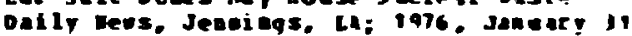

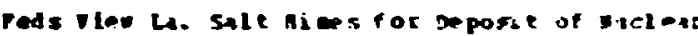
vastes

post-Signal. Cromlet, Ch: 197k, Deconber is

saclear vaste storage in $L A$. sasd olatecitet

stare tives, raton houge, LA: 747h, Dereber it

Salt rine Tasts Slate

Deus-star, Mource. LA: 197f, Deceabte it

3 L. Sizes waed tot s-voste sigit

seates-Ited. Wer orletas LA: 1976, Deceuber 29

3 La. Sites considered to sion oucled eisto

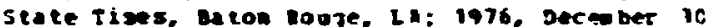

weclear instes stored in LA.?

Baraer-tribune, Pranlin, L4; 1976, noceaber $x$

L50 to Join Expadet salt nome Investipation

rhe crovley post-5ential: 197k. Hat 30

State storsqe iped for surlear Dasta

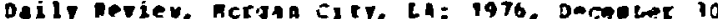

Preeport Cbevicals plans es exirace Jranido vale seape Tims. Biton lowge, LA: 1976, Decenher 2 ]

vacharie stle pooe vieved is Tose sice couet, Ibiendar, Lit, 1976, Dereeber in

Louisiand Dones Consilteret siles for spent -rul morning Adrocate. Ext on Bnuge. LA: 1976, necebder 10

Moore objects po seoring yuciant waste in sile cones

Indientor, Raseond, LA: 1976, Deceober in

Noclear sance Tests Planned in La. Miap

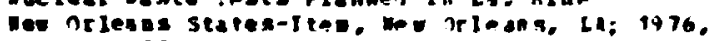
Deceeber il

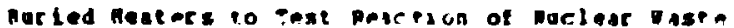
riose-plcapune, Mev orloans, LA: 197h, Deraber II

Mer Melease

molon carbide Wurleac mivision

1 -storaqu zeat stees in $6 n$ the Tices-plesuna, Unv orlathx, LA: IT7h,

neceober 2 ? 


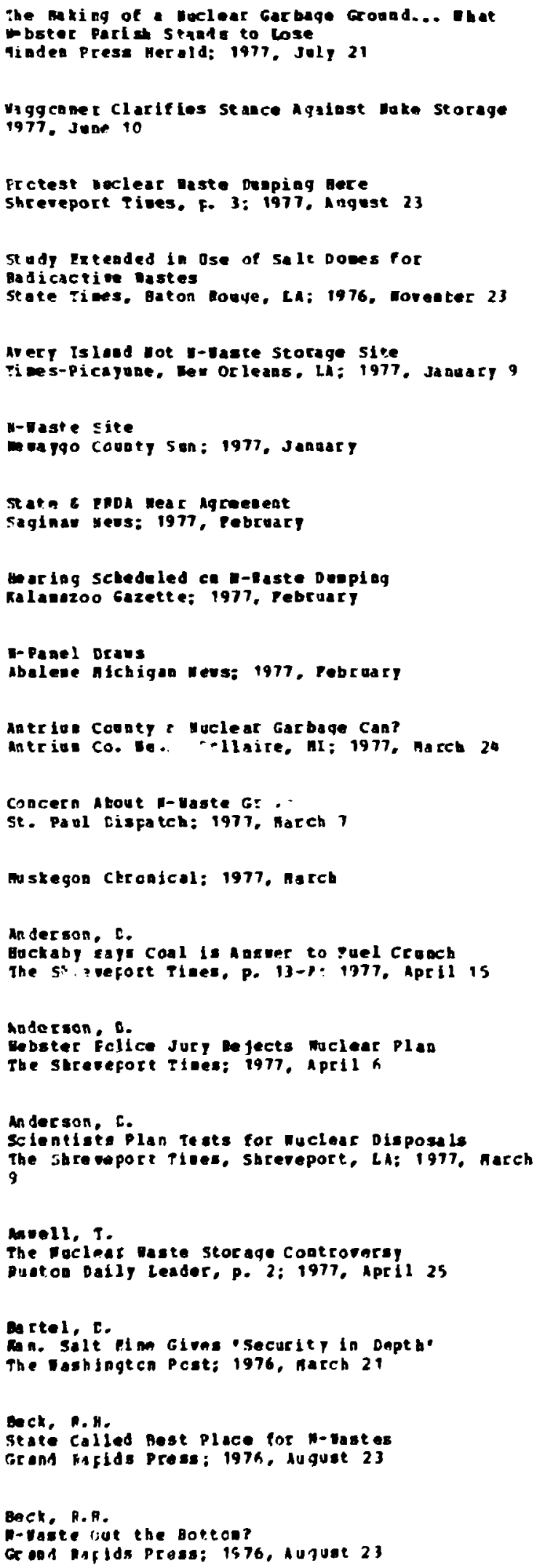


nirrete, $C$.

St. orege plat ofposed

mistor raily Leader, p. 1: 1977, April 28

rtvards. 2. (Governot, State of Lootsiane)

Sev Release

1977. Hatch 10

Geor ge. $T$.

ra the other navd...

Indian Biver Resorter. Iodian River, Michigar: 1976. Jol7 29

Ha qet, 6 .

W-iste fan Jpfosed

Ti ces-picajone, Dev Or leans, LA: 1977, April

N*ger, G.

Ficst sait Dcee oil storage slated for suener toir at

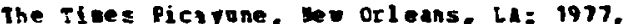

Janear $\mathbf{3}$ J

H. qer, $r$.

Hoaring ca Salt Dones Plass

Tices-picayobe. Wev Orleans, LA; 1977, Jane 2xy 11

hacris, J.

Muclear aste disposal la Parisb Discussed in

Goverace Conterence

fecord, Fiaggold, LA: 1977, Darcb 18

Hasten. $P$.

Sale mise rests not befidite ret

raily Iberion, Dev Iteria, LA: 1977, Jabarg 2

be 1doea, L.a.

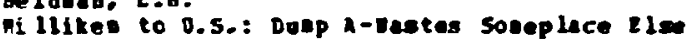

the Detrct ices press, Detroie, MI: 1977, Mat 13

Hi 11, J.

Senste iffrotes sult Doe olll

honroe Meralig varid, Bator Mogge, LA: 1977, Juge

28

ก1 11, J.

Hclear uate ba Bu Gales seate Approval

The sitrevefore tiaes: 1917, June in

Gill, J.

inste ireas

nonres, Paton souge, LA, Vol. 37, Jo. 16; 1977.

Aprili

Ai 11. J.

pe gei ci soen a berriog of m-llase is sece

The surevepore Tibes, Beton louge, li, p. 12-A;

1977, Apri1

Ai 11. J.

Muciear waste of 11 prefiled

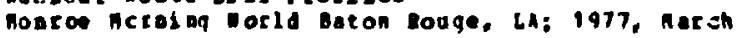
in
Ai11. J.

Ieclear Iaste storaqe Bill Avalts state

Legislators

The shreveport riees, shrevenort, LA: 1977, Harch 16

i.t1. J.

16 iree salt Dowes out As B-waste Sites

The sireveport tines, shreveport. LA: 1977.

Janeary 25

Hines, -

h-masés ieed perpetnal care

rines-Picayues, reo orleans, LA; 1977, April 5

Ror cona, L. B.

leters (teapcracy storige of hadioactive wiste)

nindes Press-herald: 1977 , April 12

HeckabT. J.

Wevs pron uashington

The bient11 e Denocrat: 1977, har 5

Aectaby. J.

vashingeos percre

Jackson Independe at, Jonesborn, L: 1977, April 23

Hotcheson, J.t.

p-naterial storage Bea Gets suppor

tíes-plcayuee, hev orleans, LA: 1977, noriI 7

Huccheson, .1. H.

HII Prohibits seorage of Muclear waste in La.

pies-Plcapune: 1977. June 11

Keneerly.

Decisios op. Mnke Inste site sot par ot

Mindea Press-Rerald, ninder, IA: 1977, April 27

rraner, h.

cautions cooperation cond he niq Liability for Gor. Al11ikea

The Sagioev Dews, Saginav, MI; 1977, June 17

Lebec, 1.

rediactive Dastes the nnsolved probless

uclear Oppoaents, p. 7: 1977. January

Lovery, R.?.

Let tar to the zditor (Mot opposed to storage of radioact ive oterials

alnden hereld-press, inden: 1977, April 19

Han ing. . H.

stetenent orging citizens to Drite their congressan C coce rolng storage of uclear uasee

id weerby sale dowes

aladan press-hera 14; 1977, hay 26

thanning, A. A.

letter to ehe enteor conponition ro hadionet ive

vace storata in Loolstana)

nindan Hersid-Press: 1977, april 27

Martin, H.: HeCoy, S.

Muclear vasto frowite prohles for 0.5.

rhe shrerepore Times, shraveport, LA: 1977, June

12 
ayo.

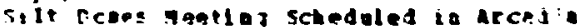

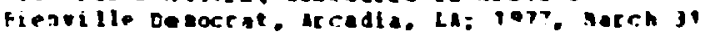

iro, T.

Liponeats Plan Mucleat Protest

bos-5116, acatoe. LA: 1977, Tap

accor. \&.

positent= Besct to M-uste proposal

Thp Shrovefort Tiees. Shreverpore, LA: 1977.

Antil za

nenitreirt, H.

sonate Mrs ecanission to control toric neterial 1777. Bit

nexinnev, 3

sertep denies state veto on welear vaste

dasbingtco J cernal: 1977, Jaly il

ni l1er. $P$.

coll tipe

Press-lietold, nindea, LA: 1977, narch 10

miller, H.

Toceriv: EBDA fisleadiag on muke Issue

niaden Press-Herald: 1977. Juae 29

miller, $n$.

Patlic Cffoses mroposal to store buclear vastes

Mintea Press-Herald: 1977, march

Mi 11s. $\mathrm{H}$.

What Georqia ofticial Dould Haw ta sep if He

Faced the laces

wucleat Opponeats, p. 5: 1975, Jalp-hugust

o.cenaor, J.

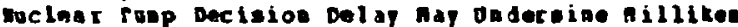

The sagtaes wos, Saginav, nI: 1977, warch 20

o.connct, J.

Mogle. inlieg want leqislatere to be able to Veto voclear busp

the nusteqon Coronicle, Nuskeqon, ar: 1977, Rerch 16

o' consor, $J$.

State and lach wear Agreenent on Iute Sate lules

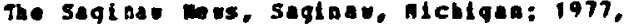

rebroary

crcennot, J.

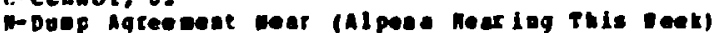

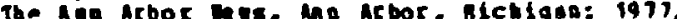

petreary 6

o' connor, J.

Hon ing schedaled on tuclear Wase dueplag ae

Al rese

Ra lasazoo fazette, Ralanozoo, Hichiqan: 1977, Petroary

a' connor, $J$.

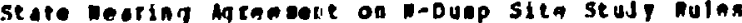

the eny ciey riees, lay City, alchinan: 1977.

pabroary
Peterson. P.

Seloin Gives gerniez on bnes viste storije

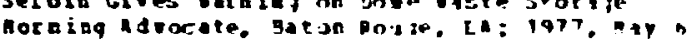

Pertis. 0.

Hoards opposed

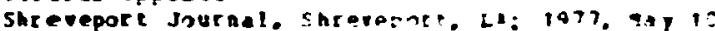

Pet is, $z$.

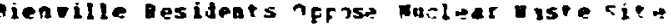

Proposal

dienville Journal: 1977, April 24

Peteis, E.

Wuclear Iaste Proble:

Shreveport Jantasl, Shreveport, LA: 1377, 9arch 11

Peteis.

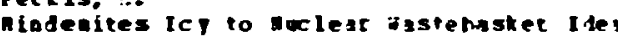

Shreveport Journal: ip77, atch 9

Pritchart. B.

leelana being lped is heo hopp 27 lecord-ragle. Traverse Citg. Michiqan: 1976 . Jelt

Shephert, P.t.

PEA Official Experes Bayou Choctio sale Done on stor aqe by ingose

stace Tibes. Euton Rougt, LA: 1477, Pebruary 17

Sholet, in.

Panel oxs D-deste singdije fan

the suravepot tiofs: 1977. Iune

steacel, 5 .

Hocleas daste is Trteky

star. Baseond, LA: 1976, necerber

Hode. $n$.

- hazardons condielons" cilel for storage of luclear vaste

Ral aoszoo Gazetife, Kalanazoo, Richigan; 1976. Recos

Dardlan, J.

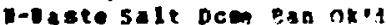

Wou Orleans stake-Iten, 9...n Ronge, LA: 1977, June 1

Delogarten, 6

nichigen Is oot is A-Duep iite - 12 other se ates on sevit Lise

onicole free Pres, Detroit, MI: 1971, July 20

Yel sะ, R.A.

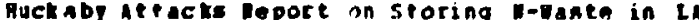

riaes plcapone. Wo or leanse LA: 1977, April 27

Will1 an, $C$

La. hay becose Wurleir nuep

Town Talk, leiand te, LA: 1977, Jenuary 13

volcote. D.R.

Mucl ant mast

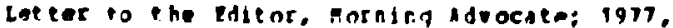
June 9 